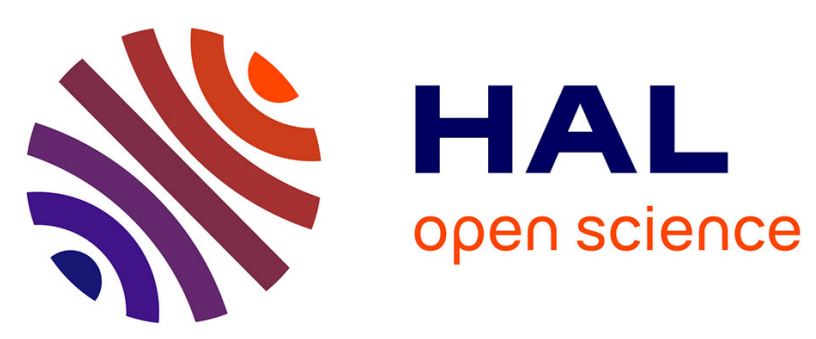

\title{
A propos de deux monuments figurés du début du $3 e$ millénaire: observations sur la Figure aux Plumes et la Prisoner Plaque
}

Camille Lecompte

\section{- To cite this version:}

Camille Lecompte. A propos de deux monuments figurés du début du 3e millénaire: observations sur la Figure aux Plumes et la Prisoner Plaque. Ilya Arkhipov, Leonid Kogan Natalia Koslova. The Third Millennium. Studies in Early Mesopotamia and Syria in Honor of Walter Sommerfeld and Manfred Krebernik, 2020, 9789004418073. hal-02471953

\section{HAL Id: hal-02471953 \\ https://hal.science/hal-02471953}

Submitted on 9 Feb 2020

HAL is a multi-disciplinary open access archive for the deposit and dissemination of scientific research documents, whether they are published or not. The documents may come from teaching and research institutions in France or abroad, or from public or private research centers.
L'archive ouverte pluridisciplinaire HAL, est destinée au dépôt et à la diffusion de documents scientifiques de niveau recherche, publiés ou non, émanant des établissements d'enseignement et de recherche français ou étrangers, des laboratoires publics ou privés. 


\section{A PROPOS DE DEUX MONUMENTS FIGURES DU DEBUT DU $3^{\mathrm{E}}$ MILLENAIRE :}

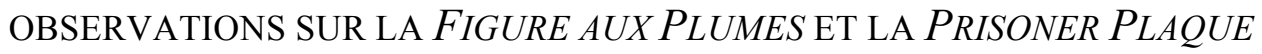

Camille Lecompte. CNRS - ArScAn - UMR 7041 - VEPMO. Nanterre.

Parmi les inscriptions lapidaires de la première moitié du $3^{\mathrm{e}}$ millénaire qui figurent sur des monuments associant texte et image, la Figure aux Plumes et la Prisoner Plaque from Kiš constituent des cas exceptionnels, la première en raison de l'hymne ou courte œuvre de genre littéraire qu'elle comporte, la seconde pour ses implications politiques et historiques ${ }^{1}$. Ces deux derniers monuments ne se rattachent de plus pas au sens strict au genre des transactions gravées sur pierre et dites kudurrus, dont certaines, d'une époque proche et tout aussi intéressantes, firent plus récemment l'objet de réévaluations, telles que la stèle d'Ušum-gal ou la Blaue Plaque ${ }^{2}$. En raison des difficultés philologiques et paléographiques inhérentes à la Figure aux Plumes et, dans une moindre mesure, à la Prisoner Plaque, dont la récente et magistrale publication par Steinkeller (2013) ouvre la voie à de riches perspectives d'études, les observations et interprétations offertes ici chercheront surtout à soumettre à la discussion des points précis.

En modeste hommage à Manfred Krebernik, l'une des figures incontournables et marquantes des études sur le $3^{\mathrm{e}}$ millénaire qui influença ma génération, je souhaite consacrer cette étude et, par là, exprimer toute ma gratitude à son encontre pour les multiples occasions durant lesquelles, généreusement et sans parcimonie de temps, il m'aida, répondit à mes questions et m'apporta d'importantes corrections, lors de discussions passionnantes et érudites au-delà même de l'assyriologie.

\section{Figure aux Plumes}

Il est bien connu que ce monument se compose de deux faces distinctes, l'une représentant un personnage pourvu d'un couvre-chef, autour duquel est disposée en lignes et cases une sorte de composition littéraire, l'autre contenant uniquement un texte divisé, de manière plus habituelle, en colonnes et cases. Bien que cette inscription soit célèbre dans la littérature assyriologique et ait fait l'objet d'études et éditions remarquables, notamment la copie de Thureau-Dangin ( $D C$, Pl. XXXIV) et la magistrale interprétation de Wilcke (1995), il m'a semblé utile ici de signaler quelques-unes des collations effectuées, voici quelques années, sur l'objet lui-même au Musée du Louvre ${ }^{3}$ et grâce à un

\footnotetext{
${ }^{1}$ Les abréviations suivent celles du RlA. Les plus fréquentes ici sont : UET 2 = Burrows $1935 ;$ ELTS = Gelb et al. 1991 ; IAS = Biggs $1974 ; D C=$ Sarzec et Heuzey 1884-1912; DA = Dynastique Archaïque. Je remercie Kamran Zand pour ses conseils, corrections et pour avoir laissé à ma disposition sa thèse. J'exprime également ma gratitude envers Grégoire Nicolet pour ses suggestions et commentaires.

${ }^{2}$ Voir ainsi Balke 2016.

${ }^{3}$ Je tiens ici à exprimer ma gratitude aux conservateurs du Musée du Louvre et du Département des Antiquités Orientales qui m'avaient apporté leur aide, particulièrement à Mme Sophie Cluzan, avec qui un projet commun sur ce même sujet est en cours et qui discuta avec moi des aspects iconographiques, ainsi qu'à Mme Béatrice André-Salvini, qui m'avait autorisé à
} 
jeu de nouvelles photographies ${ }^{4}$. L'état dégradé de l'inscription à certains endroits explique en grande partie les problèmes d'identification des signes, requérant une minutieuse observation ligne par ligne. Dans la mesure où la compréhension de cette inscription demeure en outre en partie problématique, je souhaite ajouter ici quelques commentaires, observations et suggestions afin d'enrichir la discussion sur un texte loin d'être résolu.

\subsection{Translittération}

Nous respectons, pour la face figurée, la disposition choisie dans la reconstruction textuelle dite Lesungsversuch 2 de Wilcke (1995), qui constitue l'option la plus vraisemblable ${ }^{5}$.

Face figurée

i. $\quad 0 . \quad[$ ]

1. $\quad$ sita(LAK489) $\mathrm{za}_{7}$-gin 3 ig ti

2. $\quad$ geš:nu-du ${ }_{3}$ gi nu-du 3 nam-en AK

ii. $\quad 0 . \quad[$ ]

1. mete idim? ${ }^{\text {tum }} 2$ TI

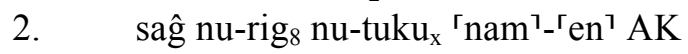

iii. 1. nam-en $\mathrm{AK} \mathrm{ša}_{3}-\mathrm{tum}_{2} \mathrm{u}_{2} \mathrm{alim}^{?}$ maš- $\mathrm{da}_{3}$ tu

2. $\operatorname{saman}_{x}\left(\mathrm{ES}_{2} \cdot \mathrm{SU}_{3} \cdot \mathrm{NUN}\right){ }^{\top} \mathrm{gid}_{2}{ }^{7 *}$-da $\mathrm{gu}_{2}$-ba DU

3. $\Gamma^{\ulcorner}$alim ${ }^{2} * 1$ tu IGI ${ }^{r} \mathrm{GI}{ }^{?}{ }^{?} *$ tu nam-en $\mathrm{AK}^{*}$

iv. 1. nam-en $\mathrm{AK} \mathrm{ša}_{3}{ }^{-}{ }^{\mathrm{T}} \mathrm{tum}_{2}{ }^{\top} \mathrm{u}_{2}$

Face inscrite.

i. $\quad 1.4\left(\right.$ bur $\left._{3}\right) \mathrm{iku} \mathrm{i}_{3}$-šum 2

2. $\quad 1\left(\right.$ sar $\left.^{\prime} u\right) \operatorname{SAR}\left(\operatorname{kiri}_{6}{ }^{3}\right)$ sanga $_{x}$

3. $5\left(\right.$ bur $\left._{3}\right)$ me-dar*

4. 3(bur 3$) i_{x}(A)$-suhur

5. 3(bur'u) NI DU

6. $\quad{ }^{\circ} 1\left(\text { bur }_{3}\right)^{\urcorner}$BE AMBAR

ii. 1. nin-ĝgir ${ }_{2}$-su diĝir

2. $\quad$ gir $_{2}$-su-me

3. uga $_{\mathrm{x}}\left(\mathrm{NAGA} \cdot \mathrm{U}_{2}\right)^{\text {mušen }} \mathrm{TAB} \mathrm{E}_{2}$-me

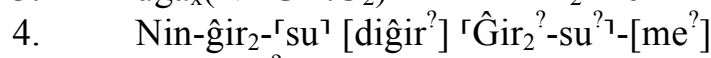

5. aša $_{5}$ kiri $_{6}{ }^{?}$ (SAR) SANGA $\mathrm{x}$ gar

iii.

1. $\quad \mathrm{TAB} \mathrm{E}_{2} \mathrm{SU}_{3}$ uga $_{\mathrm{x}}{ }^{\text {musen }}$-me

2. nin-ĝir ${ }_{2}$-su diĝir $\mathrm{su}_{3} \mathrm{C}^{\mathrm{X} \mathrm{x}^{7^{6}}}$

effectuer une première série d'observations sur l'objet. Les observations présentées ici furent rendues possibles grâce aux photographies de qualité exceptionnelle réalisées par R. Chipault.

${ }^{4}$ Le relevé accompli par Heuzey (1902) de la face figurée, qui comporte quelques détails plus justes que chez ThureauDangin, offre également un utile point de comparaison. Les références bibliographiques sur la Figure aux plumes, qui sont nombreuses, peuvent être notamment trouvées en ELTS 18 (Gelb et al. 1991 : 66) ; on y ajoutera principalement, du point de vue philologique, Wilcke 1995, ainsi que quelques allusions plus ponctuelles dans diverses publications (par exemple, Veldhuis 2006 : 193, Zand 2009 : 4 et 348, Civil 2013) et, pour une étude iconographique, Dolce 1997.

${ }^{5}$ Les collations se rapportent principalement à la copie de Thureau-Dangin mais ne concernent ni les divergences avec Heuzey 1902 ni les points déjà corrigés par Wilcke 1995 et vérifiés lors des collations.

${ }^{6}$ Selon la lecture de Wilcke $1995: 673$, on peut éventuellement y voir : ' ${ }^{\mathrm{gir}}{ }_{2}{ }^{2}-\mathrm{su}^{?}{ }^{7}-[\mathrm{me}]$. 
3. aša $_{5}{ }^{\top} \mathrm{KALAM}^{?}{ }^{7}{ }^{*}$ GI gar

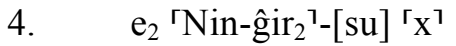

iv.
1. nam-en $\mathrm{AK}$
2. $\mathrm{u}_{4}$ tu ${ }^{\mathrm{r}} \mathrm{X} \mathrm{NIG}_{2}{ }^{? 7}$
3. $. \quad[]^{\top} \mathrm{u}_{6}{ }^{\top}-\mathrm{di}_{3}$
4. $\quad \mathrm{tut}^{?}{ }^{?} \mathrm{~T}$ igi-ĝal ${ }_{2}$
5. ${ }^{\mathrm{d}} \operatorname{lam}\left[\mathrm{ma}^{?}\right]$

V.

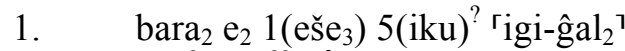

2. $\mathrm{rU}_{4}{ }^{?} \mathrm{aša}_{5}{ }^{{ }^{8} 7} \mathrm{G} \mathrm{AL}_{2}$

3. $\quad \mathrm{KUR}[\mathrm{x} x]$

4. [ ]

5. [ ]

vi.
1. $n u-{ }^{\top} x^{7}$ šu apin-la
2. $\mathrm{gal}^{*} \mathrm{du}_{11} \mathrm{za}_{3}-\mathrm{mi}_{3}$
3. nin-ĝir ${ }_{2}$-su $\mathrm{za}_{3}-\mathrm{mi}_{3}$
4. en ${ }^{\ulcorner} \mathrm{sag}^{?}{ }^{?} \mathrm{sig}{ }^{\mathrm{r}} \mathrm{MEN} \mathrm{URI}_{3}{ }^{2 * 1}$
5. [Nin]- ${ }^{\top} \mathrm{gir}_{2}-\mathrm{su} \mathrm{za}_{3}{ }^{\top}-\mathrm{mi}_{3}$

\subsection{Commentaires, observations et collations ${ }^{9}$}

\subsection{Face figurée}

i. 0 et ii. 0 : Wilcke (1995: 671-672) suppose que le début des deux premières colonnes fut, par manque de place au niveau de l'intervalle entre les masses/hampes, directement inscrit dans l'espace séparant le chef couvert de plumes et le premier de ces derniers objets à sa droite. Aucune trace d'écriture n'est d'ailleurs visible entre les premières hampes, à l'exception très incertaine d'une sorte de cassure le long de la colonne la plus à droite de la figure.

i. 2. Zand identifia la même expression dans une composition UD.GAL.NUN (Zand 2009 : 4 et 348).

ii. 1. Il nous semble que, en dépit de l'interprétation proposée par Wilcke en:me idim te, la jonction des signes ME et TE - à comparer avec l'association dans l'ordre TE ME, attestée, entre autres, dans le nom d'En-mete-na — suggère davantage de lire mete, qui, ici, signifierait, " ornement» et se rapporterait à la masse. Le signe DU pourrait être entendu comme tum ${ }_{2}^{-(m a), ~ « q u i ~ c o n v i e n t ~ », ~ s e l o n ~}$ l'association, se rencontrant apparemment surtout dans les inscriptions et textes paléo-babyloniens, entre mete et tum ${ }^{10}$. Pour IDIM, $c f$. la liste des noms de dieux : SF 1, rs. iii. 9 IDIM (LAK 4) ${ }^{11}$.

ii. 2. Nous reprenons ici la translittération proposée par Krebernik (1993/1994 : 90), mais ne saurions toutefois exclure l'interprétation de Wilcke en : sag nu-tuku $u_{\mathrm{x}}$ pa nu-tuku $\mathrm{x}_{\mathrm{x}}$, qui convient du point de vue

\footnotetext{
${ }^{7}$ Le nombre de cases dans cette colonne pourrait être de six et non cinq, ainsi que les éditions antérieures le suggèrent.

${ }^{8}$ Le signe ne ressemble pas à IGI, même si l'on attendrait à cet endroit la répétition, supposée en ELTS et par Wilcke 1995 : 673 , de l'expression igi-ĝal 2 .

${ }^{9}$ Seuls quelques passages et signes importants ou particulièrement difficiles à lire sur les photographies sont dessinés ici.

${ }^{10} \mathrm{Cf}$. par exemple : RIME E4.2.14.17, 32, mete lugal-še ${ }_{3}$ tum $_{2}$-ma.

${ }^{11}$ Krebernik $1986: 182$; Mander $1986: 86$.
} 
grammatical. Le parti ici pris suggère que cette phrase se rapporte non aux éléments inhérents à la masse - dont on ne voit d'ailleurs pas très bien pourquoi elle serait dépourvue de «tête » et de tronc ou branche - mais au fait que cette masse ne fut ni offerte ni possédée. Elle aurait alors été créée - si l'on suit la proposition ci-dessous - de manière seigneuriale sans avoir été auparavant transmise.

Le signe EN, omis par Thureau.Dangin avait déjà été repéré par Heuzey ${ }^{12}$ puis translittéré par Wilcke. ii. 2. - iii. 3. - iv. 1. L'interprétation offerte par Wilcke de l'expression récurrente nam-en AK, « tu règnes », ne nous semble pas clore les possibilités ouvertes par ce texte ambigu. La traduction par une seconde personne du singulier, bien que justifiée par la suite du texte sur la face purement inscrite et la mention de «tu es le dieu de Ĝirsu », demeure toutefois problématique. Il est d'autre part envisageable de reconstruire autrement la combinaison des signes comme : en nam-AK, « le seigneur/en l'a créé ». Cette dernière interprétation, à première vue moins convaincante, repose néanmoins sur la logique du texte lui-même, qui semble en effet se rapporter à la masse et à sa confection. En i. 2, serait ainsi marquée une opposition entre les verbes comportant une négation, insistant sur l'absence de matériau «périssable» employé pour cette masse et l'aspect seigneurial de sa création. Cette interprétation suppose néanmoins que le verbe $\mathrm{AK}$ désigne ici - de manière similaire à des textes plus $\operatorname{tardifs}^{13}$ un acte pour lequel on attend, en sumérien, $\operatorname{dim}_{2}$ - la Prisoner Plaque employant, par comparaison, MUD pour la fabrication de ce dernier objet (Steinkeller 2013 : 140). Bien qu'une telle assimilation soit discutable, l'onomastique des textes archaïques d'Ur offre une construction relativement comparable par des noms tels que Ama-hye ${ }_{2}$-AK (UET 2, PN 111), Alan-MUNUS-AK (UET 2, PN 619), ou du type Nom divin-AK (par exemple, Nanna $a_{x}$-AK, UET 2, PN 546) qui impliquent un sens tel que « créé, fait par». Comme l'a également souligné Krebernik, certains noms comportant AK et attestés dans les textes de Fāra soulèvent de semblables difficultés lexicales relatives à l'identification $\mathrm{du}$ verbe $\mathrm{AK}^{14}$. La fonction de préformative non-négative du signe NAM se rencontre ailleurs, au moins occasionnellement, dans le corpus présargonique ${ }^{15}$, mais apparemment pas avant l'époque de Fāra.

iii. 1-3. Sur le signe REC 123 = LAK 187, que Wilcke suggère d'identifier avec alim ou lulim, voir également Mittermayer (2005, 56-57), qui le compare avec ŠEG 9 et les attestations, de l'époque de Fāra, de ŠEG $9-M_{A}-D_{3}$ ( $c f$. Steinkeller 1995: 698 pour une éventuelle lecture mašda ${ }_{\mathrm{x}}$ ). La collation de la ligne iii. 3 y exclue néanmoins une interprétation en mašda $\mathrm{x}_{\mathrm{x}}{ }^{\text {mǎ́du3 }}$. Dans la mesure où l'association entre šeg ${ }_{9}$ et maš-du $u_{3}$ est fréquente dans les textes lexicaux et littéraires de l'époque de

\footnotetext{
${ }^{12}$ Ici, comme par la suite, nous nous rapportons à Heuzey $1902: 76-79$.

${ }^{13}$ Pour un emploi de AK relatif à la production de statues, cf. Renger dans le RlA 6, 309. Il est avéré que AK décrit dans des inscriptions plus tardives du deuxième millénaire la construction de temples, voir par l'inscription d'Adad-apla-iddinam, RIMB 2.8.9, 1.10 ba-ni-in-AK à propos de la construction de l'e ${ }_{2}$-bababar $_{2}$.

${ }^{14}$ Krebernik $2002: 12$.

${ }^{15}$ Sporadiquement, le signe NAM se rencontre dans des chaînes verbales telles que gu ${ }_{2}$ nam-mi-de 2 , RIME 1.9.3.1, vi. 7, «il déclara »; Hymne au Temple de Keš, vers 1 (IAS 307, i. 1-4) [na]m-[nun]-ne ${ }_{2}$ [n]am-nun-ne ${ }_{2}{ }^{\top} \mathrm{e}_{2}{ }^{\top}$-ta [na]m-ta- ${ }_{3}$, cf. Wilcke 2006, 220 et $228:$ : le caractère princier, le caractère princier le sortit du temple ». Le signe $\mathrm{NAM}_{2}$ se rencontre également dans le même emploi de préfixe na- non négatif dans les textes UGN, cf. Zand $2009:$ 24, 28.
} 
Fāra $^{16}$, une valeur ŠEG 9 de LAK 187 semble plausible. Toutefois, l'évolution graphique de LAK 187 pourrait être reconstruite, à l'instar des propositions de Wilcke, en le dérivant de UET 2, S. 163 (= alim ${ }^{17}$ ), qui consiste en un Tierkopfzeichen surmonté d'un MA ; cette même composante MA pourrait alors avoir donné la forme spécifique du signe de la Figure aux plumes.

\begin{tabular}{|l|l|l|}
\hline $\begin{array}{l}\text { Figure aux Plumes (REC } \\
123)^{18}\end{array}$ & Textes d'Uruk $^{19}$ & ED I \\
\hline Face figurée. iii. 1. & "ALIM (ZATU 26) \\
\hline Eace figurée. iii. 3.
\end{tabular}

Table 1. REC 123, signes ALIM, UET 2, S. 163 et ŠEG 9.

iii. 2. La lecture du deuxième signe comme $\mathrm{SU}_{3}$ par Wilcke (contra BU sur la copie de ThureauDangin) est confirmée. Juste en-dessous de ce signe, entre $\mathrm{GU}_{2}$ et $\mathrm{ES}_{2}$, les collations ont permis de discerner la fin d'un signe - un clou horizontal comme représenté par Thureau-Dangin — et ce qui doit être son début, consistant en un croisement de deux obliques, permettant probablement de lire, selon leur ordre de succession, $\mathrm{BU} \mathrm{GU}_{2}$ - ce dernier certes incertain selon Wilcke, mais qui semble identique à ses variantes présargoniques - L L'interprétation offerte par Wilcke ne saurait par conséquent être conservée telle quelle, le signe BU pouvant alors être associé à DA pour former gid $_{2^{-}}$ $\mathrm{da}$, tandis que $\mathrm{BA}$ correspondrait à un pronom suffixé à $\mathrm{GU}_{2}$, et ce en dépit de l'éloignement de ces signes $^{20}$. La ligne peut être comprise ainsi : $\operatorname{saman}_{\mathrm{x}}\left(\mathrm{ES}_{2} \cdot \mathrm{NUN} \mathrm{SU} \mathrm{U}_{3}\right) \mathrm{gu}_{2}$-ba gid $\mathrm{g}_{2}$-da DU « la corde sur leur nuque (i. e. des animaux mentionnés) étirée, tu/il les a(s) apporté(s) », notamment en regard des allusions, dans la littérature sumérienne, à l'aurochs tiré à l'aide de la corde saman ${ }^{21}$. Une référence à la corde de l'arpenteur (eš $\check{g i d}_{2}$-da, de manière similaire au nom de profession $\operatorname{lu}_{2}$ eš $_{2} \operatorname{gid}_{2}$-da) serait également envisageable.

\footnotetext{
${ }^{16}$ Mittermayer 2005, voir infra, pour les références ; les citations des textes UD.GAL.NUN se retrouvent en Zand $2009: 142$.

${ }_{17}^{17}$ À propos de ce signe, $c f$. Burrows dans UET 2, Pl. 13, Mittermayer $2005: 37$ et 46, Lecompte $2013: 27-28$ note 97.

${ }^{18}$ Copie de l'auteur.

${ }^{19}$ Signes provenant du site CDLI, http://cdli.ucla.edu/tools/SignLists/protocuneiform/archsigns.html.

${ }^{20}$ Dans l'ensemble, on constate que plusieurs séquences de signes suivent leur ordre de lecture, avec quelques exceptions, ce que j'ai renoncé ici à représenter dans la translittération.

${ }^{21}$ Parmi les exemples connus, citons ici la Lamentation sur Sumer et $U r$, vers 423-424 : ušumgal ka du ${ }_{8}$-a piriĝ-ĝa $\mathrm{ni}_{2} \mathrm{ni}_{2} \mathrm{il}_{2}-\mathrm{il}_{2}-$ la-bi / am dab -ba-gin $_{7}$ saman-e bi sin-šub.bu re ki-erim $_{2}$-e ba-ab-de 6 , voir Michalowski 1989, 62-63; « Leurs dragons à la gueule béante, dressés au-dessus de lions, / après qu'on leur eut passé le licol comme à un aurochs capturé, furent emportés dans des lieux hostiles $», \quad$ traduction de $\quad$ P. Attinger (http://www.iaw.unibe.ch/ueber_uns/amm_amp_va_personen/prof_dr_attinger_pascal/index_ger.html). L'image de l'aurochs (am) enchaîné et associé à une longe est une représentation courante de la littérature sumérienne.
} 

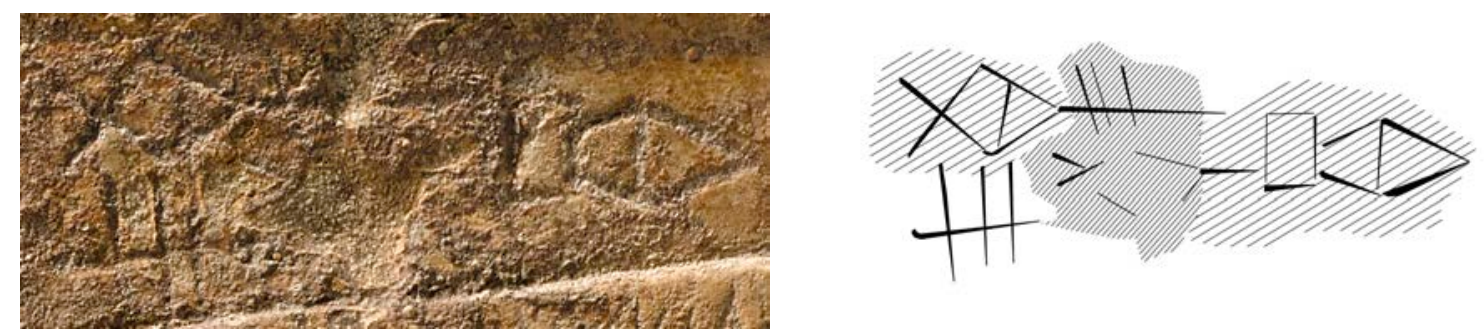

Illustrations 1. Photographie et copie de la ligne iii. 2. face figurée.

iii. 3. Le signe AK, non dessiné par Thureau-Dangin et reconstruit par Wilcke, est en fait visible, comme le montre déjà le relevé effectué par Heuzey (1902 : 76).

iii. 3. Le signe endommagé, représenté comme une simple barre horizontale par Thureau-Dangin, et

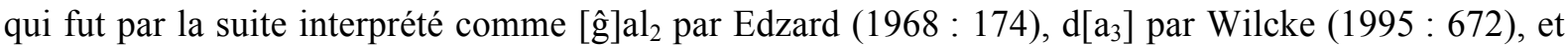
'X' par les auteurs d'ELTS (Gelb et al., 1991: 67), ne semble correspondre à aucune de ces deux premières possibilités. Selon les collations, il pourrait s'agir soit de GI, soit, moins probablement, d'un signe débutant par une forme de «tête », $\mathrm{LU}_{2}$, voire SAĜ. La reconstruction proposée par Wilcke, maš '(IGI)-[d] $\mathrm{a}_{3}$ est, quoi qu'il en soit, problématique et repose seulement sur la répétition postulée de l'expression figurant plus haut en iii. 1. S'il s'agissait de GI, je ne verrais pour le moment pas d'autre solution que de le tenir pour une graphie phonétique de šeg, s̆i-gi, à comparer avec celle adoptée dans la liste des signes d'Ebla en $\check{s}_{3} e_{3}-g u-u m .{ }^{22} \mathrm{Au}$ vu du contexte et de l'association entre alim et lulim dans

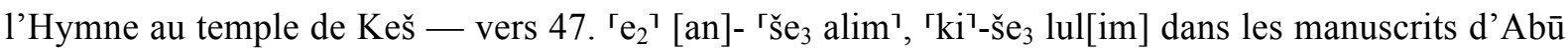
Șalābīh ${ }^{23}$-, l'on peut se demander s'il n'est pas envisageable de reconstruire, pour autant que le signe endommagé soit $\mathrm{LU}_{2},{ }^{\top} \mathrm{lu}_{2}{ }^{\top}-\mathrm{lim}$, une écriture phonétique qui serait toutefois spécifique ${ }^{24}$.
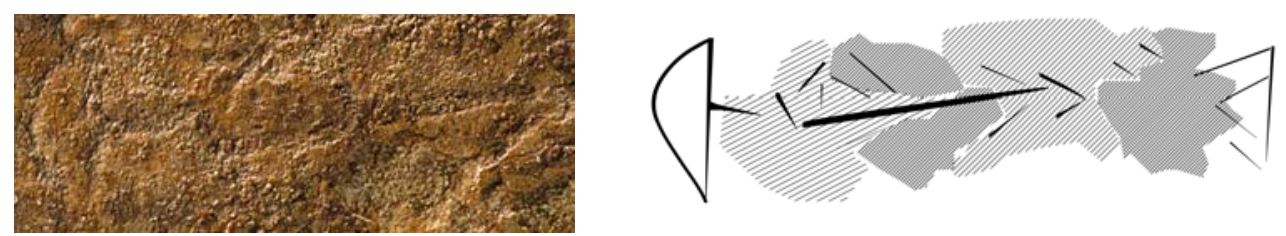

Illustrations 2. Photographie et copie de la ligne iii. 3. face figurée.

iii. 3. Le signe sur le coin gauche supérieur, non dessiné par Thureau-Dangin, s'apparente, ainsi que l'estima Wilcke, à celui figurant sur la ligne iii. 1, soit probablement ALIM ou ŠEG 9 (voir la reproduction ci-dessus).

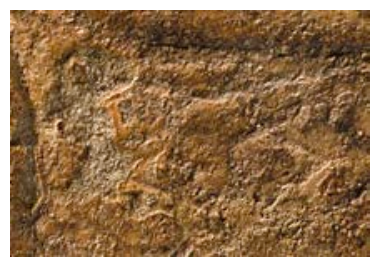

Illustration 3. Photographie de détail de la ligne iii. 2. face figurée.

\footnotetext{
${ }^{22}$ Voir ainsi Archi 1987 : 97, entrée 95. Si la suggestion de lire ši-gi s'avérait juste, le signe précédent ne pourrait être alors que alim.

${ }^{23}$ Wilcke $2006: 230$.

${ }^{24}$ En faveur d'une valeur phonétique /lim/ du signe IGI dans les textes d'Abū Șalābīh militent le signe LULIM (cf. Mittermayer $2005: 49.52$ ) et la référence au contenant bur ${ }^{\text {lim }}$ bur selon la lecture, toutefois non assurée, de Civil $1987: 144$ et 154 et Civil $2008: 72$, entrée 74.
} 


\subsection{Face inscrite.}

i. 1-6. Les surfaces agricoles énumérées sont associées à des toponymes ou des noms que l'on retrouve partiellement ailleurs, comme l'a déjà observé Wilcke : a-suhur et sanga ${ }_{\mathrm{x}}{ }^{25}$ sont ainsi attestés dans les textes de Lagaš-Ĝirsu ${ }^{26}$. Ce dernier toponyme, SAR sanga $a_{x}$, ainsi que BE AMBAR, apparaissent en outre dans la composition dite $A d$-gi $i_{4}$, comme dans des compositions UD.GAL.NUN ${ }^{27}$; BE AMBAR peut d'ailleurs être comparé avec les divers toponymes des archives et lots de textes de Lagaš-Ĝirsu comportant ce dernier signe, tels que Tir-ambar ${ }^{28}$. Au vu de l'importante surface qui lui est associée, le toponyme SAR SANGA ${ }_{x}$ 'est pas nécessairement ici un verger, bien que Civil (2013:21 et 50) le tienne pour tel dans la composition $\mathrm{Ad}_{\text {-gi }}$ et que le toponyme attesté dans les tablettes administratives de Ĝirsu en soit un. Les autres noms semblent en revanche propres à la Figure aux Plumes et ne posséder ailleurs aucun parallèle connu dans la toponymie.

i. 3. Bien que le dernier signe soit identifié par Edzard et Wilcke avec NAM, il s'avère, conformément à ce que semble montrer la copie de Thureau-Dangin, davantage similaire à DAR, notamment dans sa variante attestée dans les textes d'Ur. Le terme me-dar ${ }^{29}$ est attesté comme divinité dans les listes de Fāra et Abū Șalābīh ${ }^{30}$ ainsi que, de manière résiduelle, dans un nom propre des textes de Ĝirsu ${ }^{31}$.

Photographie du signe DAR

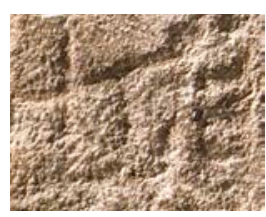

Dessin du signe DAR

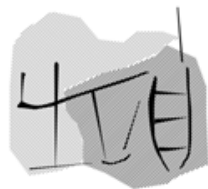

DAR dans les textes d'Ur (UET 2, S. 36)

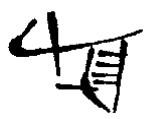

Illustrations 4. Photographie, copie du signe DAR (ligne i. 3. face inscrite), signe DAR à Ur.

ii. 4. - iii. 3. La reconstruction par Wilcke de la répétition du refrain $\mathrm{Nin}_{\text {-gir }}{ }_{2}$-su diĝir $\mathrm{Gir}_{2}$-su-me reste incertaine en raison de l'effacement des signes dont la lecture n'a pu être améliorée lors des collations. iii. 1. A propos du corbeau dans la littérature sumérienne, on se reportera à Veldhuis (2004 : 299-300), où l'on ne trouve guère de similitude avec notre inscription, si ce n'est qu'Enlil, déguisé en corbeau dans Enlil et Namzitarra, y est interpellé à la deuxième personne (uga ${ }^{\text {musen }}$ nu-me-en) ${ }^{32}$. Le thème du corbeau se retrouve pour le reste bien sûr ailleurs, notamment, dans le corpus d'Abū Ṣalābīh, en un

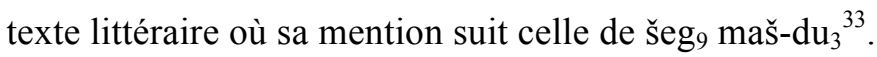

ii. 3. L'interprétation proposée par Wilcke du signe devant $\mathrm{E}_{2}$ comme $\mathrm{IA}_{2}$ (lu en ninnu) est infirmée par les collations, qui démontrent bien la seule présence de deux barres horizontales, bien que l'on

\footnotetext{
${ }^{25}$ La lecture en sanga ${ }_{x}$ telle qu'adoptée, par exemple, par Wilcke, fut conservée ici ; voir également Civil $2013: 50$ pour une discussion d'une valeur hirin ${ }_{\mathrm{x}}$ (et subsidiairement de la valeur de SAR comme déterminatif ou " verger »).

${ }^{26}$ A propos du premier, $c f$. RGTC 1, p. 208 ; pour le second, RGTC 1, p. 227, voir plus particulièrement les références à kiri $_{6}-\mathrm{i}_{\mathrm{x}}$-sanga $\mathrm{x}$.

${ }^{27}$ Veldhuis $2006: 93$; Civil $2013: 49-50$

${ }^{28}$ RGTC 1, p. 11-12; Schrakamp $2015: 205$.

${ }^{29}$ On ne peut plus retenir ici qu'il s'agit d'une référence à un prêtre išib, contra ELTS 18 et Wilcke.

${ }^{30}$ Mander $1986: 29$ (entrée 261) ; Alberti $1986: 12$.

${ }^{31}$ Selz $1995: 176$.

${ }^{32}$ Civil 1974-1977 : 68-69, vers 13-15.

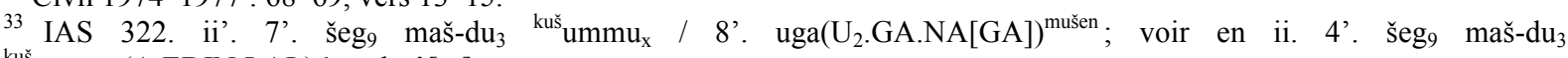
kuš $\mathrm{ummu}_{3}$ (A.EDIN.LAL) hुe $\mathrm{e}_{2}$-da- $\hat{\mathrm{g}}\left[\mathrm{a}_{2}\right]$.
} 
observe des rayures, partant sans doute du signe $\mathrm{U}_{2}$ au-dessus, débordant sur celui identifié comme TAB.

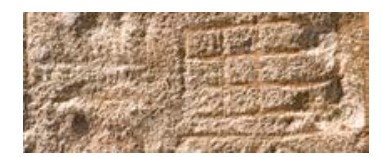

Illustration 5. Photographie de détail de la ligne ii. 3. face inscrite.

iii. 3. Le signe non identifié au milieu, qui possède, en comparaison avec la copie de Thureau-Dangin, des clous verticaux formant une boîte sur son début, rappelle quelque peu KALAM.

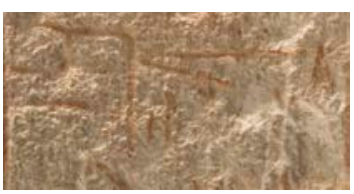

Illustration 6. Photographie de détail de la ligne iii. 3. face inscrite.

iii. 3. L'expression aša $_{5}$-- gar désigne sans doute un achat ou un type de transaction ${ }^{34}$. Dans l'inscription d'En-hुe ${ }_{2}-\mathrm{gal}_{2}$ (rev. ii. 1-3), Lugal-ki-gal / išib ${ }^{\mathrm{d}} \mathrm{Nin}^{-} \mathrm{gir}_{2}$-su /asa ${ }_{5}$ gar, se rapporte à l'acquéreur des terres mises en vente ${ }^{35}$. Cette ligne rappelle également la mention du verbe gar à la suite de surfaces, qui est compris, notamment par Marchesi et Steinkeller à propos de NTSŠ 154, comme une référence à une donation ${ }^{36}$.

iv. 4. Le signe succédant à IGI pourrait être TU.

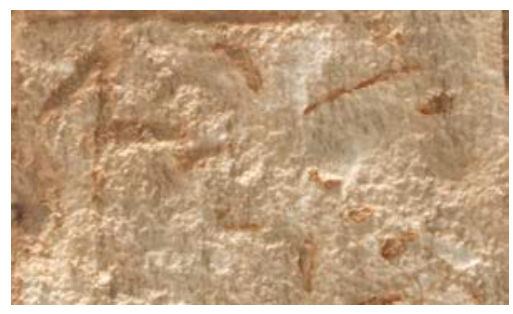

Illustration 7. Photographie de détail de la ligne iv. 4. face inscrite.

iv. 5. Après AN, il est possible de voir un signe ressemblant à KAL, par conséquent probablement lamma, même si le déterminatif est peu employé à cette époque.

v. 1. Au vu des collations effectuées, il semble exclu de voir dans le signe interprété ici, à la suite d'Edzard et d'ELTS, comme 1(eše ${ }_{3}$ ), « ein etwas mißratenes ŠITA» (Wilcke 1995: 673); la meilleure solution demeure donc de le tenir pour une référence à une surface en eše $e_{3}$, éventuellement suivie des unités dites iku, à moins qu'il ne s'agisse, comme le suggère Wilcke, du $5\left(\mathrm{IA}_{2}\right)$ possédant une valeur ninnu dans le nom du temple e $e_{2}$-ninnu. Le signe 5 semble en effet situé trop haut par rapport à l'unité eše ${ }_{3}$ et créer un décalage anormal. Une attribution au signe 5( $\left.\mathrm{IA}_{3}\right)$ de la valeur ninnu reste pour autant fragile, dans la mesure où le temple $e_{2}$-ninnu est bien représenté dans les inscriptions présargoniques par le chiffre 50 . Si l'e ${ }_{2}$-ninnu est sûrement attesté à partir du règne d'En-anatum I ${ }^{37}$, il

\footnotetext{
${ }^{34}$ Voir ainsi ELTS 20, (Gelb $\left.1991: 70\right)$; Wilcke $1996: 28$ et 30. Wilcke $2007: 67-68,82$.

${ }^{35}$ Edzard 1968: 178, estime que ĜAR doit être équivalent à $\mathrm{gu}_{7}$, alors que Diakonoff l'avait traduit littéralement en " établir », de même que Wilcke pour la Figure aux plumes : auf das Feld ... hast du gesetzt. Il est par conséquent peu probable que ĜAR y constitue une graphie défective pour $\mathrm{gu}_{7}$.

${ }^{36}$ Voir Marchesi/Marchetti $2011: 101$ note 38 ; Steinkeller $2013: 150$, note 81.

${ }^{37}$ Selz $1995: 225$.
} 
reste problématique de voir dans la Figure aux plumes sa première mention. Le terme igi-ĝal ${ }_{2}$, qui figure également dans la ligne précédente, demeure ambigu.

v. 3. Nous suivons ici Wilcke dans son identification du signe KUR : si, comme proposé par Edzard et ELTS, il s'agissait de 3(bur $\left.{ }_{3}\right)$, l'on attendrait en principe le troisième cercle (bur ${ }_{3}$ ) aligné sur le premier (juste en bas du premier signe bur 3 selon le sens de lecture de l'époque).

vi. 1. Là aussi, la combinaison des signes apin-la $a_{2}$ pourrait être rapprochée du terme désignant une location, bien que ce dernier ne soit apparemment pas attesté dans les textes provenant de Fāra et constitue l'une des catégories de terre des textes plus tardifs de la fin du $\mathrm{DA}^{38}$. Ce même terme est susceptible de se rapporter plus simplement à l'idée de culture (cf. apin-la ${ }_{2}=$ errēšsu, " laboureur, cultivateur (tenancier) »).

vi. 2. Le premier signe dans le coin, possédant apparemment des clous horizontaux sur son début, est plus probablement GAL que ME, en dépit de la copie de Thureau-Dangin et des translittérations adoptées à sa suite, notamment par Wilcke, en : $\mathrm{za}_{3}-\mathrm{mi}_{3} \mathrm{mi}_{3} \mathrm{du}_{11}$. Il semble par conséquent préférable de comprendre cette ligne comme gal $\mathrm{du}_{11} \mathrm{Za}_{3}-\mathrm{mi}_{3}{ }^{39}$, et de la traduire, avec la suivante, en " gloire à celui sur lequel sont dites de grandes choses (= au renommé), gloire à Nin-ĝirsu » ${ }^{40}$.
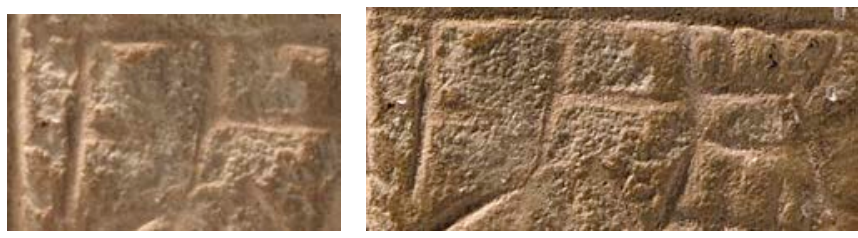

Illustrations 8. Photographies de détail de la ligne vi. 2. face inscrite.

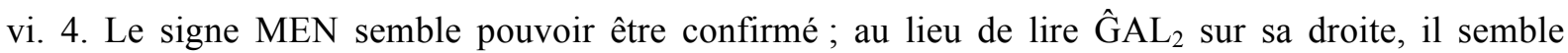
préférable de voir $\mathrm{URI}_{3}$, en raison de la présence apparente de deux barres obliques sur la partie principale du signe. La case associerait alors la couronne et l'étendard.
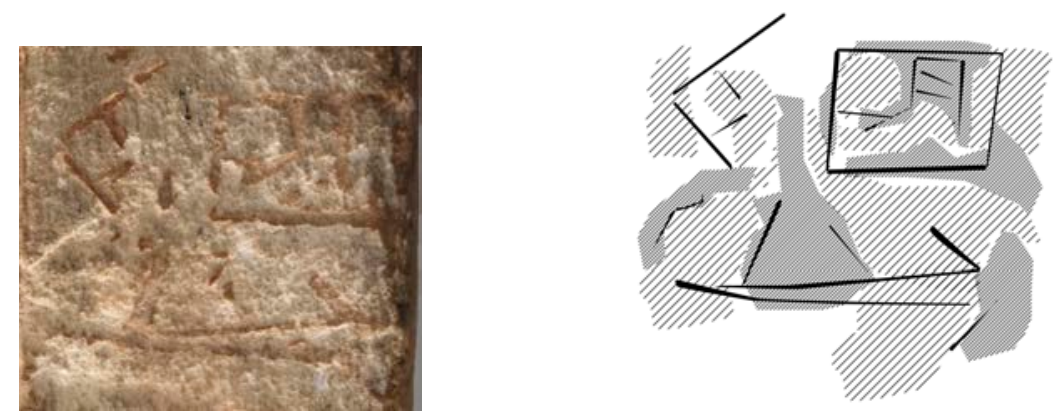

Illustrations 9. Photographie et copie de détails de la ligne vi. 4. face inscrite.

vi.5. Le signe $\mathrm{ZA}_{3}$ se devine simplement désormais.

\footnotetext{
${ }^{38}$ Voir par exemple Maekawa 1977. Un des rares points de comparaison est offert par une poignée de tablettes d'Abū Șalābīh, IAS 506, ii. 3, ainsi que IAS 539, 552-554, qui se rapportent à l'expression aša ${ }_{5}$ šuku APIN, éventuellement comparable avec le terme de Ĝirsu, mais que Krebernik (2009:20) interprète comme šuku engar, « prébende des fermiers ».

${ }^{39} \mathrm{~L}$ 'expression $\mathrm{za}_{3}-\mathrm{mi}_{3}$ gal $\mathrm{du}_{11}$ pourrait être également comparée à celle que l'on retrouve à plusieurs reprises dans l'Hymne C de Šulgi: $\mathrm{za}_{3}-\mathrm{mi}_{2}$ gal-gal du ${ }_{11}$-ga/ge du $\mathrm{du}_{7}-\mathrm{gu}_{10} \breve{s i r}_{3}-$ re-eš ${ }_{2}$ he $_{2}$-em-e-ne Voir l'édition de Castellino1972: 250 et suivantes. Attinger $1996: 757-758$, comprend différemment en : $\mathrm{za}_{3}-\mathrm{mi}_{2}$ gal-gal ka-ge du $\mathrm{g}_{7}-\mathrm{gu}_{10} \mathrm{s̆ir}_{3}$-re-eš $\breve{h}_{2}$-em-e-ne.

${ }^{40} \mathrm{Cf}$. Attinger 1996 : 510-513 sur : gal -- du $\mathrm{u}_{11} / \mathrm{di}$.
} 


\subsection{Datation, métrologie et implication historique}

Wilcke estime que la Figure aux Plumes doit être contemporaine des textes archaïques d'Ur qu'il attribue, à la suite de Karg, à la période du DA $\mathrm{I}^{41}$ : peu d'éléments grammaticaux et de préfixes verbaux y sont en effet employés, seul le préfixe nu- étant identifiable - et selon l'interprétation, certes spéculative, offerte à propos de l'expression NAM EN AK, éventuellement le préfixe nam-. On notera en revanche une possible différence vis-à-vis des textes d'Ur : conformément aux collations, la ligne iii. 2 pourrait comporter le verbe gid $_{2}$ suivi du signe DA comme marqueur du suffixe $-\mathrm{a}$, ce qui contrasterait avec une forme attestée à Ur correspondant ans doute à $\operatorname{gid}_{2}$-a, encore que Wilcke l'ait interprétée à la suite de Burrows comme un préfixe $\mathrm{a}^{-42}$. Selon le même auteur, les caractéristiques paléographiques démontrent des similitudes avec les textes archaïques d'Ur, même si les graphies de tablettes administratives et de monuments lapidaires ne sauraient se recouper entièrement en raison des différences de matériau. Les formes des signes SAĜ, présentant le même arrondi sur la «tête » que dans les textes d'Ur, ainsi que de HU, NAM et DAR, avec leur début incurvé, confortent l'hypothèse de Wilcke. En dépit de la justesse de ces observations, une date légèrement postérieure au corpus du DA I d'Ur, déjà suggérée par d'autres auteurs, tels que Marchesi et Marchetti ${ }^{43}$, qui attribuent ce monument au DA II, semble également justifiée.

Le signe numérique exprimant la plus grande surface de cette inscription ne semble en effet pas s'y conformer entièrement au système attesté dans les textes du DA $\mathrm{I}^{44}$. Si ceux correspondant à l'unité du bur ${ }_{3}$ - aux lignes i. 1, 3, 4 et 6 de la face inscrite - sont clairement identifiables, celui figurant sur la ligne i. 2 soulève quelques difficultés ${ }^{45}$. Sur la ligne 5 , les trois signes numériques se caractérisant comme des cercles doubles correspondent à l'unité bur'u (=10 bur $\left._{3}\right)$, représentée ainsi au DA I comme à l'époque de Fāra. Sur la ligne i. 2 de la face inscrite, la collation confirme bien la copie de Thureau-Dangin représentant un double cercle surmonté de 4 séries de gunûs - ou, plus concrètement, de quatre séries de deux lignes parallèles —, nous conduisant ainsi à rejeter l'identification par les auteurs d'ELTS à l'une des variantes du bur'u (= 10 bur $\left._{3}\right)$, un simple rond auxquels sont ajoutées les mêmes séries de lignes. Ce même signe, qui n'est pas attesté dans les textes archaïques d'Ur, figure avec une variante proche (les gunûs étant alors obliques) dans le système des surfaces de l'époque de Fāra avec une possible valeur du šar'u (= 600 bur $\left._{3}\right)$ d'après Krebernik (1998 : 304), bien qu'il n'y soit connu que du document TSŠ 188. Dans un premier temps, Wilcke avait suggéré d'interpréter le signe de la ligne i. 2 comme šar $_{2}$ (= 60 bur $\left._{3}\right)$, qui ne semble guère plausible, le

\footnotetext{
${ }^{41}$ Une datation des textes d'Ur du DA I a récemment été confirmée sur la base de recoupements effectués avec des échantillonnages du site, voir Benati-Lecompte $2017: 5-7,10$.

${ }^{42}$ Wilcke $1995: 670$, avec bibliographie. Plus récemment Jagersma $2010: 19$. Rappelons en outre que certains noms propres des textes d'Ur témoignent de graphies «pleines » ou continues : $\mathrm{Ni}_{2}$-kur-ra (UET 2, PN 372), Aya 2 -zi-da (UET 2, PN 46).

${ }^{43}$ Marchesi-Marchetti $2011:$ 43, 45, 91 note 318. Steinkeller $1988: 20$, avance soit le début du DA IIIa, soit la fin du DA II.

${ }^{44}$ Sur le système numérique et la métrologie des textes d'Ur, voir Burrows, UET 2, Plates 35-37 ; Chambon 2003 : 4 (pour les unités de surface) ; Friberg 1997-1998: 50-53 pour les textes comportant des mesures de champs.

${ }^{45}$ Alors que seul Edzard 1968: 175-176 interpréta l'ensemble de ces signes numériques comme des unités dénombrables calculées en bur $_{3}$ - identifiant par exemple $60(=1$ gešs 2$)$ là où il convient de lire simplement $1\left(\right.$ bur $\left._{3}\right)$ - il ne fait guère de doute, ainsi que cela ressort des éditions postérieures, que ces champs soient mesurés dans le système métrologique de surfaces.
} 
$\breve{s a r}_{2}$ étant en effet représenté par un simple cercle de taille supérieure au bur $_{3}{ }^{46}$. Wilcke corrigea plus

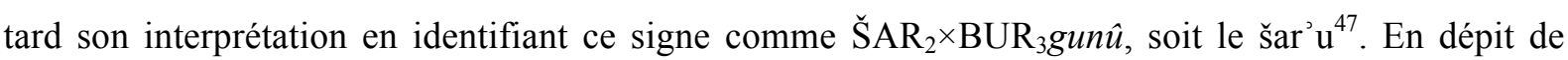
cette concordance formelle, l'écart en taille de cette surface agraire avec les autres paraît considérable. Rappelons que la deuxième superficie la plus importante, à la ligne i. 5, ne correspond guère qu'à 30 bur $_{3}$ contre 600 dans le cas qui nous intéresse. Comme, en outre, cette terre pourrait être désignée comme un verger, kiri $_{6}$, une telle surface, s'élevant alors à 3888 ha, semble par trop excessive ${ }^{48}$.

Les textes du DA I n'attestent pour leur part d'aucune variante similaire pour l'unité šar'u. Il convient cependant de rappeler que, dans le système des surfaces, il existe une incertitude sur la plus haute unité représentée : selon Burrows, il s'agirait des signes numériques $\mathrm{K}$ et $\mathrm{L}$, qu'il identifiait respectivement avec 60 et 100 bur $_{3}$, le premier étant en effet sans doute $1 \check{s ̆ a r}_{2}$, le deuxième, plus incertain, 1 šar $^{3} u^{49}$. Plus récemment, Friberg ${ }^{50}$ a toutefois estimé que, dans le document UET 2, 27, le seul attestant du soi-disant signe numérique $\mathrm{L}$, ce dernier serait en réalité identique à celui désigné comme K, soit $1 \breve{s a r}_{2}$. Dans ce cas, la seule divergence entre les systèmes d'Ur et de Fāra résiderait dans l'absence d'une variante à gunûs du bur'u, tandis que le šar'u, non attesté au DA I, pourrait très bien en théorie avoir été représenté d'une manière similaire à la Figure aux plumes. Cependant, en raison de l'absence de signes numériques à gunûs dans le corpus d'Ur, la variante connue dans notre texte peut être considérée comme plus probablement postérieure ${ }^{51}$. Il ressort en effet du système métrologique d'Ur qu'il est proche graphiquement de celui de l'époque d'Uruk, durant laquelle le šar'u semble être représenté par un signe composé de deux ronds concentriques similaire au bur ${ }^{3} u^{52}$. Les auteurs d'ELTS (Gelb et al. 1991 : 71-72) avaient déjà observé que le passage du buru représenté comme deux ronds concentriques à la forme pourvue de gunûs serait intervenue au DA I-II ; au vu du système métrologique employé à Ur, cette modification semble plus précisément attribuable au DA II.

\begin{tabular}{|c|c|c|c|}
\hline & Figure aux plumes ${ }^{53}$ & Textes archaïques d'Ur ${ }^{54}$ & Textes de Fāra ${ }^{55}$ \\
\hline 1 bur'u & (1) & $\bigcirc_{\text {H et J }} \bigcirc_{\text {signes }}$ & (ㅇ \\
\hline
\end{tabular}

\footnotetext{
${ }^{46}$ Rappelons qu'Edzard 1968 : 174, avait également assimilé ce même signe à 3600, soit 1( šar $_{2}$ ).

${ }^{47}$ Wilcke $2004: 444$.

${ }^{48}$ Une identification avec un šar 2 , soit 388,8 ha, ou un bur' ${ }^{3}$, soit 64,8 ha, parait de ce point de vue moins invraisemblable.

${ }^{49}$ UET 2, 27. rs. iii. 1.

${ }^{50}$ Friberg 1997/1998 : 51 ; Chambon $2003: 4$ semble avoir arrêté ses diagrammes sur les unités de surface au buru (à la fois les signes $\mathrm{H}$ et $\mathrm{J}$ ).

${ }^{51}$ Wilcke 2004 : 444, mentionne ainsi la présence de signes numériques pourvu de gunû dans la tablette dite d'En-he ${ }_{2}-$ ĝal $_{2}$, qu'il date du DA II, bien que d'autres auteurs l'assignent à la période de Fāra, voir ainsi ELTS 20 (Gelb et al. 1991: 69) et Steinkeller $1988: 20$, position qui semble plus vraisemblable en raison des formes angulaires et généralement non arrondies de signes tels que $\mathrm{LU}_{2}$. Cette cohérence entre les deux textes nous semble confirmer une datation de la Figure aux plumes du DA II, postérieure aux textes d'Ur.

${ }^{52}$ Voir le texte MS 4558, translittéré sur CDLI (P006327) et qui juxtapose les unités bur 3 (N14), buru (N50), šar 2 (N45), šar u (N50) et šargal (N63).

${ }_{53}^{5} \mathrm{D}$ 'après Thureau-Dangin, $D C \mathrm{Pl}$. XXXIV.

${ }^{54}$ Signes pris de UET 2, Plate 37.

${ }^{55}$ Krebernik $1998: 304$
} 


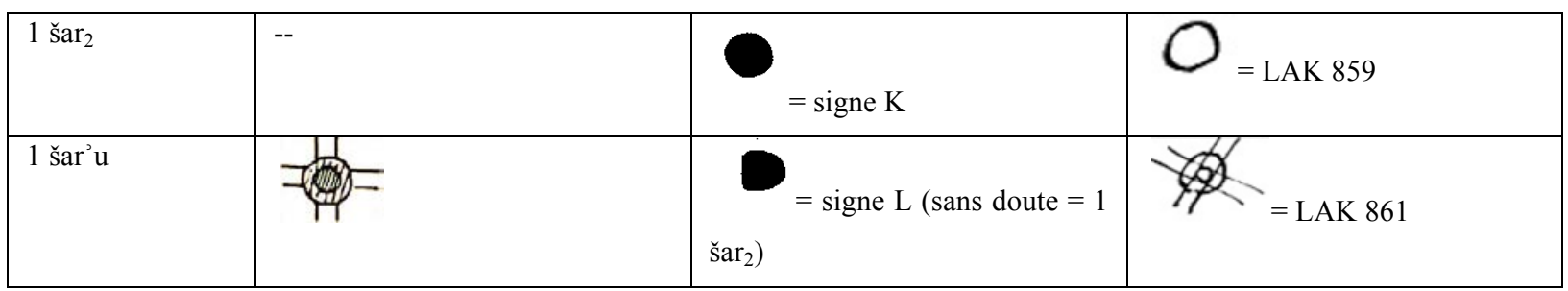

Table 2. Comparaison des unités de surface dans la Figure aux plumes, les textes archaïques d'Ur et ceux de Fāra.

La mention du temple de Nin-ĝirsu, sous la forme de $\mathrm{e}_{2}$-Ĝir ${ }_{2}$-su — et non comme $\mathrm{e}_{2}$-ninnu, qui semble discutable - semble en constituer la première attestation écrite et témoigne, avec d'autres documents, de son importance avant l'installation de la Ière Dynastie de Lagaš. Un petit lot de huit tablettes administratives datant de l'époque de Fāra et retrouvées sur le Tell K (à $12 \mathrm{~cm}$ sous le Sol d'Ur-Nanše), sur le site de Telloh, reflètent également de possibles activités cultuelles à une date postérieure à notre inscription ${ }^{56}$. En outre, la masse d'arme de Mesilim rapporte que ce dernier souverain entreprit des travaux en faveur de ce même dieu ${ }^{57}$ qui aboutirent à une modification du complexe qui lui est consacré. Si l'antériorité de la Figure aux Plumes sur l'époque de Fāra ne saurait être précisément déterminée au vu des connaissances actuelles, les analyses portant sur la stratigraphie de l'e $e_{2}$-ninnu, à vrai dire compliquées par les maigres données publiées par les fouilleurs, mirent en évidence des phases remontant au plus tôt, apparemment, au DA II, sans parvenir toutefois à faire ressortir l'existence d'une phase d'intervention de grande ampleur antérieure au règne de Me-silim ${ }^{58}$. Bien que l'existence du DA II demeure un sujet débattu ${ }^{59}$, l'attribution à cette période de la Figure aux Plumes, soutenue par divers auteurs, concorde avec les observations d'ordre métrologique. Ce monument serait par conséquent légèrement postérieur aux textes archaïques d’Ur, rédigés vers 2750/2700, mais antérieur au règne de Me-silim, étant donc situé, de manière imprécise, dans cet intervalle, encore que la paléographie tende à privilégier une proximité avec ceux-là. Une datation du — début du - DA II s'avère en outre cohérente avec l'existence d'un corpus, limité en nombre, de tablettes principalement administratives qui semblent témoigner d'une étape intermédiaire entre les documentations du DA I et de l'époque de Fāra ${ }^{60}$. Même si, à Ur, cette phase du DA II ne semble pas exister, la transition entre les périodes du DA I et IIIa soulève diverses incertitudes pour un intervalle qui dura sans doute un siècle. La Figure aux plumes nous semble par conséquent pouvoir y appartenir.

\footnotetext{
${ }^{56}$ Deux d'entre elles (RTC 7 et 8) se réfèrent en effet à des offrandes à des divinités qui doivent être liées aux activités cultuelles du temple de Nin-ĝirsu. Krebernik $1998: 374$ avec bibliographie.

${ }^{57}$ RIME 1.8.1.1, 4, où le souverain affirme avoir construit le temple de Nin-ĝirsu.

${ }^{58}$ On pourra comparer ainsi les reconstructions stratigraphiques proposées par Forest 1999, Huh 2008 et Marchesi-Marchetti 2011. Ces derniers (2011 : 38-44) identifient ainsi un niveau datant du DA II (phase 1) et deux autres indistinctement attribués au DA II/IIIa (phases 2 et 3), sans toutefois préciser clairement leurs arguments pour cette date. Huh 2008: 141, 150 ne suggère pas de datation aux différentes unités stratigraphiques qu'elle distingue, mais les objets servant de repère à Marchesi-Marchetti (2011) permettent de faire coïncider les SE 4-5, Komplex I, SH, avec une période antérieure à Me-silim. Forest $1999: 15$, estime que les phases antérieures au règne de Me-silim ne sont pas suffisamment documentées et que les parties anciennes de la Construction Inférieure seraient attribuables à ce même souverain. Rappelons en outre que, sur le Tell $\mathrm{K}$, des strates antérieures au DA furent bien entendu mises au jour, mais sur des secteurs différents et après les fouilles de Sarzec et Heuzey, $c f$. Huh $2008: 130-131$.

${ }^{59}$ Marchesi-Marchetti $2011:$ 4-5, note 16, Benati-Lecompte $2017: 6$.

${ }^{60}$ Parmi les vingt-cinq textes récemment réédités ou édités par Krebernik et al. 2015, neuf semblent dater d'une époque intermédiaire (Textes $1,5,7,9,12,13,15,19,20,21)$, tandis que dix s'apparentent aux textes d'Ur $(3,6$, peut-être 10, 11, $14,16,17,18,19,23)$, le reste étant soit postérieur (2), soit antérieur $(24-25)$, soit incertain $(4,8,22)$. On peut ajouter aux textes « intermédiaires » du DA II un fragment de Nippur (AS 17, 4).
} 


\subsection{Quelques perspectives d'interprétation}

La Figure aux Plumes, connue pour soulever d'importantes difficultés de compréhension ${ }^{61}$, a été interprétée par Wilcke comme un texte littéraire ${ }^{62}$. En dépit de légères divergences exprimées ailleurs $^{63}$, il ne saurait faire de doute qu'une partie de ce texte ne se rattache pas au genre des transactions de terre ni des contrats, notamment en raison des parallèles avérés dans des textes littéraires, plus particulièrement la composition $\mathrm{Ad}_{\text {-gi }}$ et le corpus UD.GAL.NUN. Wilcke estima par ailleurs que cette inscription constituerait un document «juridique» qui traiterait d'un acte de donation, ou, plus probablement, d'une attribution de terre, voir d'une charte d'immunité valable pour les champs figurant sur la face inscrite ${ }^{64}$. Or, comme vu ci-dessus, l'expression aša ${ }_{5}$-- gar se rapporte vraisemblablement à un achat - littéralement aux champs « posés »-, tandis que l'identification de apin-la $\mathrm{a}_{2}$ pour la «location» nous semble très douteuse. Dans ce cas, et compte tenu de l'absence de noms de personnes qui correspondraient aux vendeurs et acheteurs, il est envisageable que la doxologie marquée par la répétition, sur le revers, du terme $\mathrm{za}_{3}-\mathrm{mi}_{3}$ introduise l'acquisition et la constitution - même progressive — d'un domaine agraire appartenant au temple de Nin-ĝirsu. Un tel domaine aurait pu être d'ailleurs attribué au dieu tutélaire de Ĝirsu à l'occasion de la (re)fondation de son temple, constituant un octroi dont on ne sait qui fut à son origine. Le total des surfaces des champs, notamment à la ligne i. 2 de la face inscrite avec le possible šar ${ }^{3} u$, qui atteindrait 4166,40 hectares, représente un ensemble agricole réaliste pour une institution religieuse qui devait occuper une place centrale dans l'organisation de la cité ${ }^{65}$. C'est pourquoi le terme aša ${ }_{5}$-- gar pourrait ici caractériser, à défaut d'une acquisition dont on ne trouverait ici trace du prix, la confirmation de l'intégration des terres mentionnées dans le domaine de Nin-ĝirsu ou un transfert immédiat, qui préfigurerait d'une certaine manière le but que s'assignaient les Réformes d'Iri'inimgina, marquant la propriété divine sur un ensemble de champs ${ }^{66}$. Il est également intéressant de noter que cette même expression semble structurer une partie du texte du revers, dans la mesure où elle paraît associée à deux noms qui font certainement écho à la liste des champs : si la première mention, aša $a_{5}$ SAR

\footnotetext{
${ }^{61} \mathrm{Cf}$. Gelb et al $1991: 66:$ « If there is anything certain that can be said about this text, it is that we understand nothing about it ».

${ }^{62}$ Voir entre autres Zand $2009: 32$; Benati-Lecompte $2017: 25$

${ }^{63}$ Civil 2013 : 17 note 10 : «More realistically, the inscription seems to be a kudurru-like monument to piously celebrate the purchase or possession of certain tracts of land by an anonymous ruler or the assignation of some fields to Ningirsu ». Rubio 2009,34 , considère également notre monument comme « essentially an early land-sale document ».

${ }^{64}$ Wilcke 1995: 671 ; Wilcke $1996: 5$, suggère soit une donation (Stiftung) soit une mise en fief (Belehnung); Wilcke 2007 : 26 ; Cavigneaux 1998, 74.

${ }^{65}$ Par ordre de comparaison, rappelons que l'importance du domaine agraire du temple de Ba- $\mathrm{U}_{2}$ fit l'objet de spéculations, notamment de la part de Deimel qui était parvenu à une évaluation de 4500 hectares ( $c f$. Selz 1995 : 40 pour les références bibliographiques).

${ }^{66}$ Cela rejoint l'idée de Civil $2013: 17$ note 10 (voir ci-dessus), sans toutefois dénier à ce monument un caractère littéraire. Il est bien connu que, dans les Réformes d'Iri'inimgina, ce dernier souverain proclame Nin-ĝirsu propriétaire des champs de $l^{\prime} \mathrm{e}_{2}-\mathrm{mi}_{2}$, dans un contexte fort différent de celui de la Figure aux plumes. Toutefois, il est possible que ce document manifeste une appropriation d'un domaine que le dieu et son temple revendiquent et s'assimilent. Dans ce cas, l'on trouverait ici une expression de l'idéologie à laquelle Iri'inimgina prétend s'affilier.
} 
$\mathrm{SANGA}_{\mathrm{x}}$ gar, correspond à la deuxième terre agraire dans l'ordre de succession, les autres sont sans doute sous-entendues sous la deuxième apparition, $a^{2}{ }^{2}{ }_{5}{ }^{~} \mathrm{KALAM}^{?\urcorner} \mathrm{GI}$ gar.

Même si la face inscrite réitère l'expression AK NAM EN ainsi que, sans doute, le refrain : « Nin-ĝirsu, tu es le dieu de Ĝirsu », il nous semble exister, principalement dans les thématiques, une certaine rupture relevée par Wilcke entre les deux côtés, qui se manifeste d'ailleurs matériellement par l'absence de tout signe cunéiforme et de continuité sur les tranches. Ce même auteur avait, du reste, déjà repéré les principales caractéristiques des deux parties de l'inscription. La face figurée, marquée par une sorte de refrain en AK NAM EN et la répétition d'éléments qui semblent, selon son interprétation, relatifs à la faune - mais pas de maš-da $a_{3}$ mentionné une seule fois - et aux prairies, s'avère donc purement littéraire. La face inscrite, mêlant doxologie et références à des champs, est en revanche plus complexe et d'autant plus ambigüe qu'une surface apparemment plus réduite de 1 eše ${ }_{3}$ et $5 \mathrm{iku}$ ( ?) (=3,96 ha) serait également mentionnée plus loin dans les colonnes cassées. Un point qui semble également incertain concerne la focalisation de l'inscription: bien que Wilcke ait traduit l'ensemble du texte à la deuxième personne, à l'exception de la doxologie, les seuls marqueurs clairs se trouvent sur la face inscrite sous la forme de : -me-(en). Il n'est par conséquent pas certain que le reste soit concerné, la face figurée pouvant être comprise dans son ensemble à la troisième personne.

Dans ce monument, les relations entre image et texte s'avèrent également complexes, l'analyse iconographique et l'identification des éléments étant en partie débattues. Dans le cadre du présent article, je me borne à résumer les résultats des recherches de $\mathrm{S}$. Cluzan qui seront développées ultérieurement dans une étude conjointe et systématique ${ }^{67}$. Les nervures du couvre-chef du personnage sur la face figurée, interprétées à l'origine comme des plumes puis réévaluées par divers auteurs comme une ornementation végétale, présentent en réalité de fortes similitudes avec la représentation des ailes de l'Anzu de monuments de la fin du DA. Le couvre-chef pourrait donc bien s'avérer être composé de plumes. Un autre élément important est l'identification des trois sortes de hampes ou piquets qui se trouvent sur la droite de personnage : contrairement à une opinion courante ${ }^{68}$, il ne s'agit probablement pas de trois masses identiques, la ligne la plus à droite, brisée sur sa partie supérieure, correspondant en effet plus vraisemblablement à la limite d'un espace défini. Les deux hampes, surmontées d'objets arrondis qu'elles dépassent, sont bien des poteaux soutenant des masses, puisque celles retrouvées en Mésopotamie et datant du $3^{\mathrm{e}}$ millénaire possèdent des caractéristiques similaires,

\footnotetext{
${ }^{67}$ Une analyse préliminaire fut présentée lors de la conférence : «Sur quelques aspects de la Figure aux Plumes », tenue lors de la RAI 61, à Berne le 25 juin 2015. Les conclusions exposées ici sont évidemment sujettes à débat : ainsi, la nature des deux ornements composant le couvre-chef, dont nous estimons qu'elles sont bien des plumes, fut diversement interprétée, Marchetti, dans Marchesi-Marchetti 2011 : 190, note 20 et 195, ayant supposé qu'il s'agissait d'orge, hypothèse s'appuyant sur d'autres représentations mésopotamiennes. Une autre interprétation, qui ne suit malheureusement pas la même rigueur et commise par Silvia Schroer (OBO 88, p. 171-172), aboutit à des analyses pour le moins contestables, et à tout dire superficielles et ineptes : l'auteur compare en effet, sans solution de continuité, les éléments couvrant le chef du personnage avec des représentations où seraient, selon elle, figurées des feuilles de palmier, mais provenant d'aires géographiques bien différentes, notamment du Levant ou d'Assyrie, et postérieures, pour certaines, de plus d'un millénaire et demi ! Silvia Schroer semble en outre identifier avec une déesse (!!) le personnage au buste nu de la Figure aux plumes, qui porte pourtant une barbe clairement identifiée dès sa publication en $D C$, mais étonnamment omise par ce même auteur sur son dessin.

${ }^{68}$ Par exemple $D C$, p. 165 ; Wilcke $1995: 669$.
} 
étant ainsi souvent perforées de part en part ${ }^{69}$, argument auquel s'ajoute l'association entre Nin-ĝirsu et ce type d'arme ${ }^{70}$. Par ailleurs, le schéma iconographique d'un poteau ou d'un étendard dédoublé marquant un espace central qu'il délimite et définit est également bien connu dans le monde des images des $4^{\mathrm{e}}$ et $3^{\mathrm{e}}$ millénaires. L'espace ainsi encadré est en réalité le lieu que l'image entend révéler, les symboles surmontant ou accompagnant les poteaux venant probablement identifier sa nature, voire l'identité de celui à qui il est lié. Cette caractéristique permettrait ainsi de concevoir que le personnage principal, éventuellement le dieu Nin-ĝirsu lui-même (ou son représentant) ${ }^{71}$, se présente face à un bâtiment que l'on identifierait comme son temple, inscrivant ainsi-la recension des terres agraires dans son domaine et vers lequel il tendrait la main, s'il s'avérait qu'il ne saisit pas l'un des poteaux.

\section{La Prisoner Plaque from Kiš}

Dans la mesure où Steinkeller (2013) en offrit une édition exemplaire, seuls quelques points de détail sur cet objet récemment porté à la connaissance des assyriologues seront ici discutés ${ }^{72}$.

\section{1. Commentaires ponctuels}

i. 3'. et passim. L'identification du signe GIŠIMMAR dans la séquence l'associant à ĝeš kiri $_{6}$ semble quelque peu incertaine. Alors que, dans les textes d’Ur, GIŠIMMAR ressemble déjà en grande partie aux formes attestées à l'époque de Fāra (UET 2, S. 124), aucune des variantes connues à Uruk ne se rapporte sûrement à celle observable ici. Dans tous les cas de figure, le signe est en effet fermé sur son extrémité droite. Si seule, d'après la liste de signes archaïques établie sur le site CDLI ${ }^{73}$, la variante d'Uruk dite GIŠIMMAR ${ }_{\mathrm{a} 3}$ est ouverte, encore que ses deux derniers traits soient horizontaux et non recourbés vers le haut, l'unique attestation la concernant apparemment ne permet de confirmer cette forme et semble au contraire identique à GIŠIMMAR ${ }_{\mathrm{a} 2}{ }^{74}$. Le signe figurant sur la Prisoner Plaque s'apparente toutefois, par la série de quatre traits sur sa partie gauche - souvent obliques à Uruk, mais horizontaux dans ce monument, comme dans l'une des variantes de l'époque Uruk $\mathrm{IV}^{75}$ - ainsi

\footnotetext{
${ }^{69}$ L'hypothèse de Cavigneaux 1998 : 74, qui suggérait d'identifier ces objets avec des vases, ne semble par conséquent guère convenir ; voir, à propos de l'identification des masses, Wilcke $1995: 669$.

${ }^{70}$ Il suffira ici de rappeler que Nin-girsu est représenté tenant une masse dans la Stèle des Vautours; de même, l'arme šar ${ }_{2}$ $\mathrm{ur}_{3}$ de ce dieu a-t-elle pu être identifiée avec une masse.

${ }^{71}$ Dolce 1997 : 2-3, avait estimé plus vraisemblable d'identifier le personnage de la Figure aux plumes avec le «chef de la communauté » qu'avec un dieu: selon elle, ce souverain, représenté avec un emblème plus tard transféré au divin, se révèlerait ici le «garant du culte divin et de l'ordre naturel ». Marchesi-Marchetti 2011 : 195, 212, note 7, avec références bibliographiques, prennent en revanche parti en faveur d'une identification avec le dieu qui est célébré dans l'inscription, position qui nous semble plus convaincante.

${ }^{72}$ Déjà mentionné en Civil $2003: 51$ note 9 . Je remercie K. Zand de cette indication. Même si N. Marchetti exprima un doute sur l'authenticité de cette plaque (communication personnelle relayée dans Benati-Lecompte $2017: 26$, note 75), 1'inscription ne saurait être tenue pour un faux. Du reste, l'iconographie de la scène, dont Steinkeller a démontré la cohérence avec les représentations de Kiš, tend à conforter l'authenticité des figures; on trouve, en outre, des arcs composites relativement similaires à ceux portés par l'une des figures de la plaque dès l'époque d'Uruk.

${ }^{73}$ Lien: http://cdli.ucla.edu/tools/SignLists/protocuneiform/archsigns.html.

${ }^{74}$ Tablette inédite, mais accessible sur le site du CDLI, P004291, O0104. Pour le reste, signalons que, dans la liste des signes archaïques établies en ZATU, deux autres formes — dont les attestations ne sont pas indiquées — sont également ouvertes, sous la catégorie $\mathrm{b}$. En outre, une forme de la catégorie a, sur la droite, semble également commencer de manière parfaitement identique au signe de la Prisoner Plaque, mais est malheureusement brisée sur sa moitié droite et doit correspondre à GIBIL. Dans le répertoire des signes d'Uruk, GIBIL (ZATU 214) présente d'ailleurs de manière surprenante davantage de ressemblance avec notre signe, mais est exclu en raison de la présence de NE dans cette même inscription.

${ }^{75}$ ATU 5, W 9578,p. O0101.
} 
que par la forme de cercle s'ouvrant et rempli de lignes se croisant, à la variante dite GIŠIMMAR ${ }_{a 1}$, à l'exception toutefois de sa fermeture et de la présence de deux traits obliques sur partie droite. Bien qu'une telle suggestion ne contribue en rien à éclaircir le texte, voire même l'obscurcisse, le signe en question nous semble davantage similaire au $\mathrm{GAR}_{3}$ de l'époque des textes d'Ur et de Fāra. Une attestation d'un verger de palmiers ne semble par ailleurs pas plus assurée dans les tablettes du DA en général $^{76}$.

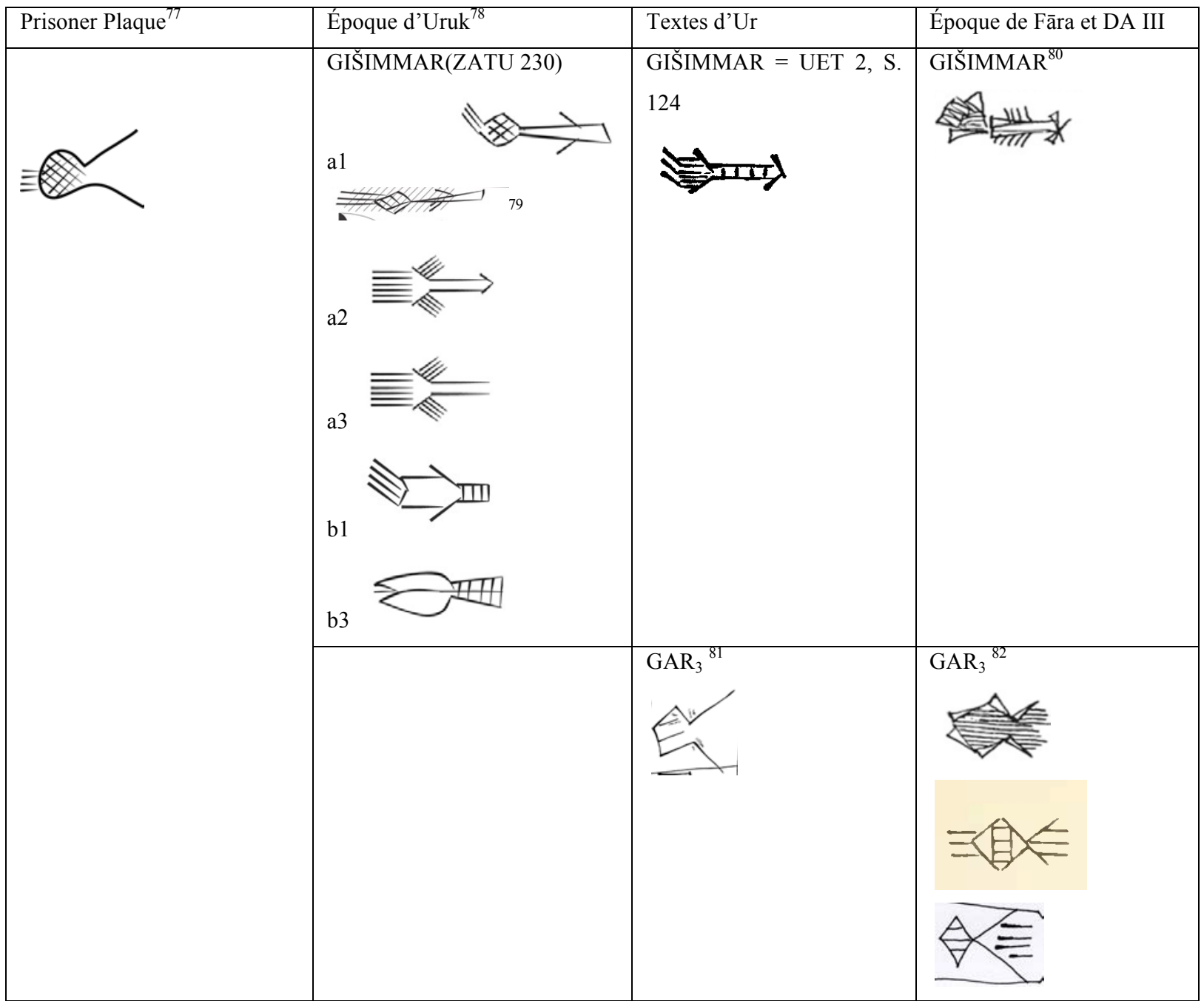

Table 3. Le signe GIŠIMMAR de la Prisoner Plaque comparé aux variantes d'Uruk, d'Ur et de Fāra ainsi qu'au signe GAR .

i. 14. BAR RA AN KI. Si le KI est bien un déterminatif, ce nom peut être comparé avec bar-ra-an ${ }^{\mathrm{ki}}$ des textes sargoniques de Gasur, confortant les localisations de Steinkeller dans le nord-est de l'Irak actuel $^{83}$

\footnotetext{
${ }_{77}^{76}$ Les attestations postérieures de palmeraies désignées comme ${ }^{\hat{g} e \check{s}}$ kiri ${ }_{6}$ g gešnimbar sont évidemment plus courantes.

${ }^{77}$ Dessin de l'auteur.

${ }_{78}^{78}$ Signes provenant de la liste du site CDLI, voir ci-dessus.

${ }^{79}$ ATU 5, pl. 061, W 9578,p = P001247.

${ }^{80}$ D'après LAK 196

${ }^{81}$ ATFU 2, O0101.

${ }^{82}$ Respectivement LAK 160 ; signe REC 95 employé dans l'inscription provenant de Nippur d'Ur-Enlil (AnNip. 1) ; inscription de Mari (IX E 48 SE 9).

${ }^{83}$ Référence en Schrakamp $2015: 233$.
} 
i. 15'. Les signes numériques semblent identiques à celui dessiné en v. 5', en raison de la présence d'une sorte de clou horizontal sur leur gauche, mais aussi de leur taille, nettement supérieure à celle du signe représentant le chiffre $10=1(\mathrm{u})$. Ces signes peuvent par conséquent être considérés comme correspondant à l'unité šar 2 (3600). Si la ligne i. 15' se réfère bien, alors, à 18000 prisonniers, le total atteint par les cases préservées, à l'exception de vi. 3', correspondant selon Steinkeller au total (36 000), s'élève à 46 920. Si l'on considère v. 5' (avec 6300 prisonniers) comme un sous-total et qu'on la retranche du reste, l'on obtient en tout 40620 prisonniers dans les cases préservées. En additionnant v. 5' et vi. 3', les deux dernières cases se rapportant à un nombre de prisonniers, l'on obtient $6300+36$ $000=42300$. Ce total correspondrait éventuellement à l'ensemble des entrées et impliquerait qu'il manque 280 prisonniers dans les cases non conservées, mais, comme mentionné ci-dessous, la reconstruction du nombre en ii. 2' reste problématique.

Illustrations 10 : signes numériques en i. 15' :

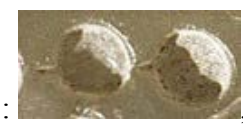
différents de $10=1(\mathrm{u})$, (ici ligne iv. 7').

ii. 2'. Le seul signe numérique sûrement visible est 60 ; il semble difficile de reconstruire la séquence numérique selon la proposition de Steinkeller en $600+60 \times 2$.

ii. 9'. DIŠ LUL:ki:da. La toponymie d'Ebla atteste d'un nom éventuellement homonyme, lu-la-du, qui pourrait indiquer une lecture lul-da ${ }^{\mathrm{ki}}$ (RGTC 12, p. 223 et 402).

ii. 11. MUŠ $\check{S}_{3}$ est incertain.

iii. 3. EZEN $\times I G I / B A$ SUM. Si l'on exclut une identification avec $E Z E N \times B A D_{3}$, le premier signe peut être comparé avec EZEN $\times \mathrm{SIG}_{7}$ (i. e. IGIgunûs), dont la lecture kisig est donnée par des listes lexicales plus tardives ${ }^{85}$. S'il semble inconnu à Fāra et Ur, on le trouve néanmoins apparemment dans une liste fragmentaire d'Abū Șalābīh (IAS 29, ii. 6'). Le signe ŠUM ${ }_{2}$ reste certes incertain, mais pourrait constituer un complément phonétique en $\operatorname{sig}_{10}$.

iii. 7'. DIŠ hal: ${ }^{[m a}{ }^{?}$ T. MA est sûr d'après la photographie. Le nom peut être comparé avec ha-la-am ${ }^{\mathrm{ki}}$, attesté dans les textes sargoniques d'Ešnunna, ce qui s'accorde de la situation géographique de l'inscription ${ }^{86}$.

iv. 4'. NI KA×ŠSU, lu en $\mathrm{i}_{3} / \mathrm{u}_{\mathrm{x}}$-bum par Steinkeller. NI peut avoir la valeur ${ }^{~} \mathrm{a}_{5} ; \mathrm{KA} \times \breve{\mathrm{S}} \mathrm{U}$ peut être aussi tenu pour $\mathrm{su}_{12}{ }^{87}$.

iv. 6'. DIŠ La-la-um. La toponymie d'Ebla offre un autre point de comparaison avec le possible homonyme la-la-mu (RGTC 12, p. 215 et 401).

iv. 14. DIŠ ur 4 :na:a. La lecture $\mathrm{ru}_{\mathrm{x}}$ de $\mathrm{ur}_{4}$ à l'époque de Fāra, et plus particulièrement dans la liste géographique d'Abū Ṣalābīh et Ebla ${ }^{88}$ permet de lire le toponyme peut en : na-a-ur ${ }_{4}\left(\mathrm{ru}_{\mathrm{x}}\right)$, na-ur ${ }_{4}\left(\mathrm{ru}_{\mathrm{x}}\right)-\mathrm{a}$.

\footnotetext{
${ }^{84}$ Source photographique : CDLI, P451403.

${ }^{85}$ cf. MSL XV, Diri IV, 100, p. 151 et N $8: 08$, p. 32 ; MSL XIV, VIII, 97, p. 479.

${ }^{86}$ Schrakamp $2015: 229$ pour les références et un rejet de l'identification avec Alep proposée par Sommerfeld.

${ }^{87}$ cf. Bauer, St.Pohl 9, p. 295.

${ }^{88}$ Kebernik 1998 : 297.
} 
v. 2'. PIRIĜ KALAM. Davantage qu'à PIRIĜ, dont la «tête », sur sa partie gauche, est remplie, depuis les textes d'Ur, voire depuis l'époque d'Uruk, de clous verticaux et non, comme c'est le cas ici, horizontaux, ce signe laisse songer à l'une des variantes de « KIŠ » (S. 161-b dans UET 2), que l'on retrouve notamment dans la séquence similaire à celle dite $\mathrm{EREN}_{2}+\mathrm{X}$, sans qu'une identification avec ce lieu ne soit assurée ${ }^{89}$.

v. 3'. Bil $1_{x}($ UET 2 S. 377+NE)-kalam. Le signe sous NE n'est pas à proprement parler DU, mais S. 377 dans UET 2, un précurseur de ĜEŠ. Il pourrait s'agir simplement d'un nom propre Bil $1_{\mathrm{x}}$-kalam, à comparer avec Bil $\mathrm{x}_{\mathrm{x}}-\mathrm{kalam}-\mathrm{du} \mathrm{u}_{10}$ des textes de Fāra ${ }^{90}$.

vi. 3'. Mes-nun dumu-lugal. Plutôt que d'y voir un prince, dumu-lugal, terme en tant que tel peu attesté — puisque l'on rencontre davantage la succession NP fils de NP2, lugal/ensi ${ }_{2}{ }^{91}$ —, cette case pourrait se rapporter à un nom propre, lugal-banda 3 -mes-nun, voire deux, les éléments mes-nun et lugal-banda $a_{3}$ étant en effet plus ou moins bien documentés dans l'anthroponymie ${ }^{92}$. Le seul anthroponyme dont le statut social est indiqué semble être celui qualifié de $\mathrm{u}_{3}$-gal-gal.

vi. 11. DIŠ LAK131 KALAM. Deimel suggérait de comparer le signe LAK 131 avec celui, figurant dans la liste des dieux, translittéré en $\mathrm{UR}_{3}$ BARgunû, équivalent de $\mathrm{Ur}_{3}$-bar-tab ${ }^{93}$ et apparaissant dans le terme AB.TAB.BAR ${ }^{94}$.

\subsection{Datation, discussion de quelques caractéristiques et perspectives historiques}

Steinkeller mit déjà en valeur plusieurs caractéristiques paléographiques de la Prisoner Plaque qui constituent des points de comparaison avec les textes $\mathrm{d}^{9} \mathrm{Ur}^{95}$ et le conduisirent à dater le monument de la même période, à savoir, selon lui, du DA II. Tout comme pour la Figure aux Plumes, les signes numériques contribuent à une discussion sur la situation chronologique de ce monument. Ainsi, les chiffres les plus élevés sont-ils à nouveau représentés par des formes inconnues des textes d'Ur: le šar’u, identique à la forme de la Figure aux Plumes, est similaire à celle, standard, employée à Fāra pour l'unité de surface, tandis que le šar 2 , un cercle précédé d'un clou horizontal, n'est apparemment pas attesté sous cet aspect à Ur et constitue une variante spécifique. Cette observation s'avère

\footnotetext{
${ }^{89}$ Lecompte 2014.

${ }^{90}$ Pomponio $1987: 61$.

${ }^{91}$ On peut ainsi rappeler que Me-ane-si, fils d'En-anatum I, se présente, dans la statue qu'il voue, selon ce schéma, voir RIME 1.9.4.15, i. 3-6; il en va de même de Lumma-tur, autre fils d'En-anatum I, dans la transaction de terre le concernant, cf. ELTS 22, i. 6-9.

${ }_{92}$ Le nom Lugal-banda 3 apparaît au DA III, voir Andersson 2012 : 106 et 312 pour son interprétation ; Mes-nun-(ne $)_{2}$ ) s'avère en revanche plus rare, étant principalement mentionné dans le manuscrit de la Liste Royale Sumérienne publié par Steinkeller, ce qui conforterait l'identification proposée par ce dernier entre les deux noms propres, voir les références en Steinkeller $2013: 139$.

${ }_{93}$ Krebernik $1986: 203$; Krebernik $1998: 283$; Mander $1986: 43$ et 161.

${ }_{94}^{94}$ Identifié comme un nom de profession en IUO VI/A p. 109 et WVDOG 143, p. 169.

${ }^{95}$ On pourra ajouter ici également la forme arrondie de certains signes, plus particulièrement de $\mathrm{GAR}_{3} / \mathrm{GIŠIMMAR}_{\mathrm{S}} \mathrm{BAR}$, LAK 131, et, dans une moindre mesure, des composés de KA, qui tranchent avec une tendance à dessiner de manière plus angulaire durant l'époque de Fāra. Il est intéressant d'observer que les signes UM, MES et DUB semblent également conformes aux usages d'Ur : ainsi, DUB, dans dub-sar sur le revers, ressemble à la forme de UM des textes de Fāra, UM en iv. 6' consiste en une boîte de signes obliques entrecroisés, et MES possède une forme également spécifique. Ces variantes ne sont toutefois pas entièrement identiques avec celles connues à Ur (UET 2, S. 77 et 297), $c f$. Benati/Lecompte $2017: 16$ note 35 .
} 
cohérente avec d'autres particularités de l'inscription dont Steinkeller estime qu'elles ne s'accordent pas avec les graphies archaïques, du moins de l'époque d'Uruk, plus particulièrement certains signes postérieurs et l'emploi de ligatures cohérentes avec les textes d'Abū Șalābīh. On peut y ajouter la présence du signe $\mathrm{UR}_{4}$, auquel Krebernik suggérait d'attribuer une valeur $\mathrm{ru}_{\mathrm{x}}$, notamment dans les graphies de noms géographiques des $\mathrm{LGN}^{96}$. L'usage des déterminatifs semble complexe : si, selon Steinkeller, les noms de lieux en sont dépourvus tandis que kiri ${ }_{6}$ et les noms divins sont respectivement précédés de geš et de diĝir, il nous semble que la présence de cinq signes KI dans les toponymes préservés ne permet d'écarter définitivement une valeur non syllabique de ce dernier. Du reste, l'irrégularité de la notation du déterminatif est manifeste en d'autres documents, plus particulièrement des listes de toponymes de l'époque d'Uruk ${ }^{97}$. Ce constat vaut notamment pour BAR AN KI, qui pourrait se lire bar-an ${ }^{\text {ki }}$ (voir ci-dessus) et éventuellement lul-da ${ }^{\text {ki98 }}$. Puisque les textes d'Ur furent rédigés entre 2750 et 2700 (DA I à Ur), la Prisoner Plaque doit dater d'une époque légèrement postérieure au plus tôt. Comparée au style plus archaïque de la Figure aux Plumes, elle semble néanmoins quelque peu plus moderne, mais les différences régionales ne permettent de les mettre sur le même plan.

Il est en revanche plus surprenant de constater que les signes numériques décrits ci-dessus ne paraissent pas plus concorder avec ceux des textes de Fāra, dans la mesure où la variante du šar’u en vi. 4 y est employée dans les unités de surface. Ce genre de graphie se retrouve en revanche dans des tablettes plus tardives de la fin de l'ère présargonique, par exemple d'Umma. Si l'archaïsme de la plaque ne saurait a priori autoriser une datation postérieure à l'époque de Fāra, le système de dénombrement pourrait s'expliquer à la rigueur comme l'expression d'une particularité régionale anticipant l'usage de signes numériques plus tardifs. La provenance nord-babylonienne de cette plaque tout comme le fait qu'elle soit gravée sur pierre pourraient tout aussi bien expliquer la permanence de traits archaïques, telles les particularités graphiques similaires aux textes d'Ur, dans un contexte plus tardif. Les points communs avec les manuscrits de la liste géographique d'Abū Ṣalābīhn, l'aspect «moderne » du système numérique ainsi que la notation de déterminatifs justifieraient alors d'une rédaction au tout début de l'époque de Fāra ou à la fin du DA II.

\begin{tabular}{|l|l|l|l|l|}
\hline & Prisoner Plaque & Textes d'Ur & Époque de Fāra & DA IIlb \\
\hline 10 & - & - & 0 & 0 \\
\hline 60 & & $\square, \square$ & $D$ & $\square$ \\
\hline
\end{tabular}

\footnotetext{
${ }^{96}$ Voir ci-dessus.

${ }^{97}$ Voir ainsi les textes dits Geography 3-4 (ATU 3, p. 161) et MS 3173, encore en partie inédit, voir sa translittération sur le site du CDLI (P252184), édition en préparation par l'auteur.

${ }^{98} \mathrm{La}$ valeur du signe KI dans les autres toponymes, LA KI BA/IGI, BA/IGI HI A et [x š]a KI est plus incertaine.
} 


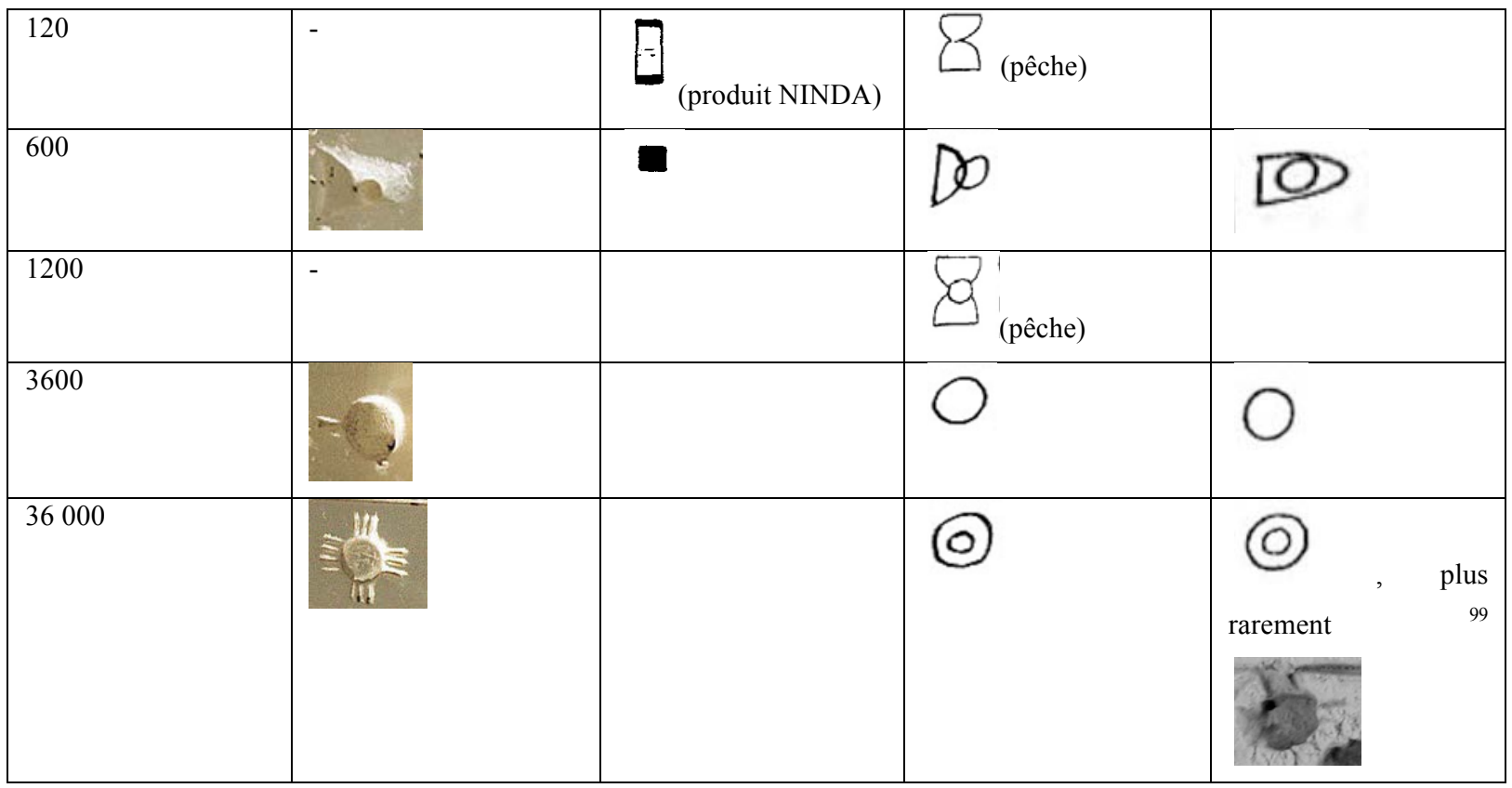

Table 4. Comparaison des unités de dénombrement dans la Prisoner Plaque, les textes des périodes du DA I, de Fāra et du DA IIIb.

Un autre aspect qui peut être brièvement discuté concerne l'organisation du texte et des cases : si Steinkeller estime que, à l'exception des lignes v. 1'-2' et vi. 1'-2', la séquence: toponyme nombre de prisonniers prévaut, l'on peut se demander si l'inverse, qui semble plus commun dans la documentation cunéiforme, ne s'appliquerait pas ici. Dans la colonne i, il pourrait ne manquer qu'une case au début répondant au schéma suivant : n šaga / ğě̌ kiri ${ }_{6}$ G[IŠIMMAR ( ?)] / DIŠ TUM, soit un nombre de prisonniers suivis de leur attribution, à savoir un verger, qui s'applique aux autres cas, puis le toponyme. Le début de la colonne v serait plus inhabituel : [n šaga] / DIŠ « PIRIG » KALAM / DIŠ ${ }_{\text {ĝeš }}$ kiri $_{6}$ GIŠIMMAR ( ?) Bi1 $1_{\mathrm{x}}$-kalam / DIŠ Šubur, soit : « $\mathrm{n}$ prisonniers, toponyme 1 pour le verger $\mathrm{X}$ (sous la direction de) $\mathrm{Bil}_{\mathrm{x}}$-kalam, (et) toponyme $2 »$. Suivrait alors un premier sous-total, incluant une liste de possibles officiels chargés des prisonniers. Sur la colonne vi, le début pourrait constituer le prolongement de la fin de la colonne v, tandis que les lignes vi. 4'.5' correspondraient à un second sous-total de 36000 - la relecture de i. 15' aboutissant désormais à un total de prisonniers supérieur à 36000 - suivi de leur emploi, si-si sur ${ }_{12}$ tab-tab $z^{2}{ }_{3}$, « to fill the threshing floors (with grain) and to make grainstacks » selon Steinkeller.

Ce dernier auteur ayant déjà parfaitement traité des implications historiques de cette inscription, notamment pour le rôle de Kiš ${ }^{100}$, il n'est guère besoin de réitérer ni commenter ici ses propos, si ce n'est pour légèrement nuancer l'idée d'un déclin de Kiš lors du DA IIIa : la datation suggérée ici, légèrement postérieure aux textes d'Ur et plus proche de l'époque de Fāra, concorderait

\footnotetext{
${ }^{99}$ CUSAS 23, 46 rs. ii 3, se rapportant à une quantité d'orge.

${ }^{100}$ On mentionnera ici que Frayne 2009 avait également fait le point sur le rôle politique de Kiš lors de la période du DA I-II et les relations entre les principaux protagonistes de cette époque.
} 
avec divers indices déjà présentés par Marchesi ${ }^{101}$ - allusion des textes de Fāra à une possible hégémonie de Kiš, nom du roi Lugal-Utu attesté à Mari et Kiš — en faveur du maintien du pouvoir de cette cité.

\section{Références bibliographiques}

Benati-Lecompte 2017 Benati, G. ; Lecompte, C. Nonadministrative Documents from Archaic Ur and from Early Dynastic I-II Mesopotamia: A New Textual and Archaeological Analysis. JCS 69 : 3-31.

Alberti 1985

Alberti, A. A Reconstruction of the Abū Șalābīkh God-List. SEL 2: $3-$ 23.

Andersson 2012

Andersson, J. Kingship in the Early Mesopotamian Onomasticon. 2800-2200 BCE. Uppsala.

Attinger 1996

Attinger, P. Eléments de linguistique sumérienne. La construction de

Archi 1987 $d u_{11} / e / d i$ «dire » (OBO, Sonderband). Fribourg, Suisse-Göttingen.

Balke 2016 Archi, A. The "Sign-list" from Ebla. Eblaitica 1: 91-113.

Balke. T. The Interplay of Material, Text, and Iconography in Some of the Oldest "Legal” Documents. Balke. T.; Tsouparopoulou, C. (éd.). Materiality of Writing in Early Mesopotamia (Materiale Textkulturen 13). Berlin-Boston. Pp. 73-95.

Burrows 1935 Burrows, E. Archaic Texts (UET 2). Londres.

Castellino 1972

Cavigneaux 1998

Castellino, G. Two Šulgi Hymns (BC) (SS 42). Rome.

Chambon 2003

Civil 1974-1977

Civil 1987

Civil 2003

Civil 2008

Cavigneaux, A. Un detail de la Figure aux Plumes. NABU 1998 : 73.

Chambon, G. Archaic Metrological Systems from Ur. CDLJ 2003:5.

Civil, M. Enlil and Namzitarra. AfO $25: 65-71$.

Civil, M. The Early History of HAR-ra: The Ebla Link. Cagni, L. (éd.): Ebla 1975-1985. Naples. Pp. 131-158.

Civil, M. Of Bows and Arrows. JCS 55 : 49-54.

Civil, M. The Early Dynastic Practical Vocabulary A (Archaic HARra A) (ARES 3). Rome.

Civil 2013

Civil, M. Remarks On AD-GI 4 (A.K.A. "Archaic Word List C" or “Tribute"). JCS $65: 13-67$

Dolce 1997

Dolce, R. Aux origines de la royauté à Tello. Akkadica 103 : 1-5.

Edzard 1968

Edzard, D. O. Sumerische Rechtsurkunden des III. Jahrtausends aus der Zeit vor der III. Dynastie von Ur (ABAW 67). Munich.

\footnotetext{
${ }^{101}$ Marchesi dans Marchesi-Marchetti 2011 : 101.
} 
Forest 1999

Frayne 2009

Friberg 1997-1998

Gelb et al. 1991

Heuzey 1902

Huh, 2008

Jagersma 2010

Krebernik 1986

Krebernik 1993/1994

Krebernik 1998

Krebernik 2002

Krebernik-Postgate 2009

Krebernik et al. 2015

Lecompte 2013

Lecompte 2014

Maekawa 1977

Mander 1986
Forest, J.-D. Les premiers temples de Mésopotamie (BAR International Series 765). Oxford.

Frayne, D. R. The Struggle for Hegemony in "Early Dynastic II" Sumer. The Canadian Society for Mesopotamian Studies 4 : 37-75.

Friberg, J. Round and Almost Round Numbers in Proto-Literate Metro-Mathematical Field Texts. AfO 44/45 : 1-58.

Gelb. I.; Steinkeller, P. ; Whiting, R. M. Earliest Land Tenure Systems in the Near East: Ancient Kudurrus (OIP 104). Chicago.

Heuzey, L. Musée National du Louvre, Catalogue des Antiquités Chaldéennes. Paris.

Huh, S. K. Studien zur Region Lagǎs. Von der Ubaid- bis zur altbabylonischen Zeit (AOAT 345). Münster.

Jagersma, B. A Descriptive Grammar of Sumerian. Thèse. Leiden.

Krebernik, M. Die Götterlisten aus Fāra. ZA 76 : 161-204.

Krebernik, M. Compte-rendu de ELTS. AfO 40-41 : 88-91.

Krebernik, M. Die Texte aus Fāra und Tell Abū Ṣalābīhn. Attinger, P. ; Wäffler, M. (éds.). Mesopotamien. Späturuk-Zeit und Frühdynastische-Zeit (OBO 160/1). Fribourg, Suisse-Göttingen. Pp. 235-427.

Krebernik, M. Zur Struktur und Geschichte des älteren sumerischen Onomastikons. Streck, M. P. ; Weninger, S. (éds.). Altorientalische und semitische Onomastik (AOAT 296). Münster. Pp. 1-74.

Krebernik, M. ; Postgate, J. N. The Tablets from Abu Salabikh and Their Provenance. Iraq $71: 1-32$.

Krebernik, M.; Steible, H.; Yıldız, F. Pr.-Fāra-zeitliche Texte aus Fāra. Koslova, L. et al. (éd.). Studies in Sumerian Language and Literature: Festschrift für Joachim Krecher (Babel und Bibel 8). Winona Lake, IN. Pp. 327-82.

Lecompte, C. Archaic Tablets and Fragments from Ur. From L. Woolley's Excavations at the Royal Cemetery (Nisaba 25). Messine.

Lecompte, C. Suggestions and corrections to ATFU - 3. Again on EREN2+S. 161b ${ }^{\mathrm{ki}}$. CDLN $2014: 22$.

Maekawa, K. The Rent of the Tenant Field (gán-APIN.LAL) in Lagash. Zinbun $14: 1-54$.

Mander, P. Il Pantheon di Abū-Sālabīkh. Contributo allo studio del pantheon sumerico arcaico (IUO Series Minor 26). Naples. 
Marchesi-Marchetti 2011

Michalowski 1989

Mittermayer 2005

Rubio 2009

Pomponio 1987

Sarzec-Heuzey 1884-1912

Schrakamp 2015

Selz 1995

Steinkeller 1988

Steinkeller 1995

Steinkeller 2013

Veldhuis 2004

Veldhuis 2006

Wilcke 1995
Marchesi, G.; Marchetti, N. Royal Statuary of Early Dynastic Mesopotamia (MC 14). Winona Lake, IN.

Michalowski, P. The Lamentation over the Destruction of Sumer and Ur (MC 1). Winona Lake, IN.

Mittermayer, C. Die Entwicklung der Tierkopfzeichen. Eine Studie zur syro-mesopotamischen Keilschriftpaläographie des 3. und frühen 2. Jahrtausends v. Chr. (AOAT 319). Münster.

Rubio, G. Chapter 2. Sumerian Literature. Ehrlich, C. S. (éd.). From an Antique Land : an Introduction to Ancient Near Eastern Literature. Plymouth. Pp. 11-75.

Pomponio, F. La prosopografia dei testi presargonici di Fara (SS, NS3). Rome.

Sarzec, E. de et Heuzey, L. Découvertes en Chaldée. Paris.

Schrakamp, I. Geographical Horizons of the Presargonic and Sargonic Archives. Sallaberger, W. ; Schrakamp, I. (éds.). ARCANE III. History and Philology. Turnhout. Pp. 197-270.

Selz Untersuchungen zur Götterwelt des altsumerischen Stadtstaates von Lagaš (OPSKF 13). Philadelphie.

Steinkeller, P. Grundeigentum in Babylonien von Uruk IV bis zur frühdynastischen Periode II. Jahrbuch für Wirtschaftsgeschichte : 1127.

Steinkeller, P. Review of M. W. Green and H. J. Nissen, Zeichenliste der archaischen Texte aus Uruk. BiOr 52 : 689-713.

Steinkeller, P. An Archaic "Prisoner Plaque" from Kiš, RA 107: 131157.

Veldhuis, N. Religion, Literature and Scholarship: the Sumerian Composition Nanše and the Birds with a Catalogue of Sumerian Bird Names (CM 22). Leiden-Boston.

Veldhuis, N. How Did They Learn Cuneiform? Tribute/Word List C as an Elementary Exercise. Michalowski, P. ; Veldhuis, N. (éds.). Approaches to Sumerian Literature. Studies in Honour of Stip (H. L. J. Vanstiphout) (CM 35). Leiden-Boston. Pp. 181-200

Wilcke, C. Die Inschrift der "Figure aux plumes"- ein frühes Werk sumerischer Dichtkunst, in Beiträge zur Kulturgeschichte Vorderasiens: Festschrift für Rainer Michael Boehmer, U. Finkbeiner, R. Dittmann et H. Hauptmann (éds.), 669-74 
Wilcke 1996

Wilcke 2004

Wilcke 2006

Wilcke 2007

Zand 2009
Wilcke, C. Neue Rechtsurkunden der Altsumerischen Zeit. ZA 86: 167

Wilcke, C. ED Lú A und die Sprache(n) der archaischen Texte. van Sodt, W.H. (éd.). Ethnicity in Ancient Mesopotamia. Papers Read at the $48^{\text {th }}$ Recontre Assyriologique Internationale Leiden, 1-4 July 2002. Leiden. Pp. 430-445.

Wilcke, C. Die Hymne auf das Heiligtum Keš. Zu Struktur und "Gattung" einer altsumerischen Dichtung und $\mathrm{zu}$ ihrer Literaturtheorie. Michalowski, P. ; Veldhuis, N. (éds.). Approaches to Sumerian Literature. Studies in Honour of Stip (H. L. J. Vanstiphout) (CM 35). Leiden-Boston. Pp. 201-237.

Wilcke, C. Early ancient Near Eastern law : a history of its beginnings : the early dynastic and Sargonic periods. Winona Lake, IN

Zand, K. Die UD.GAL.NUN-Texte. Ein allographisches Corpus sumerischer Mythen aus dem Frühdynastikum. Thèse de doctorat, Iéna. 